\title{
Rechtswörter und Rechtsbilder in den paulinischen Briefen.*
}

Von Otto Eger, Professor des römischen Rechts an der Universität Basel.

Von den Stellen, in welchen Paulus Verhältnisse des menschlichen Lebens und ihre Regelung durch das menschliche Recht zum Vergleich heranzieht, führt auf das Gebiet des Erbrechts, Gal. 3, $15 \mathrm{ff}^{1}{ }^{1}$

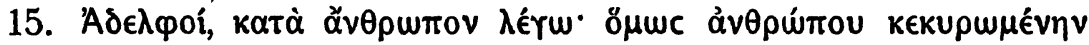

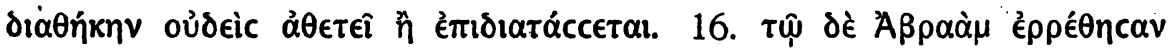

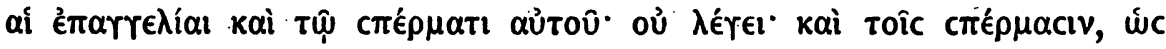

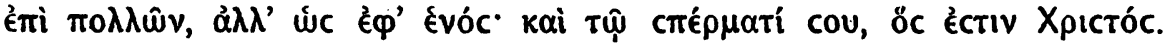

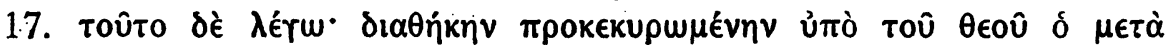

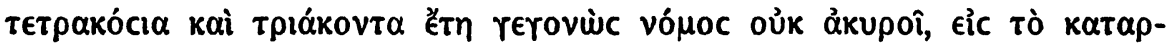

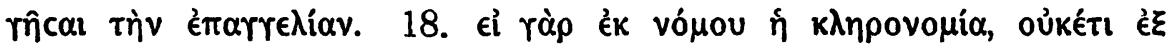

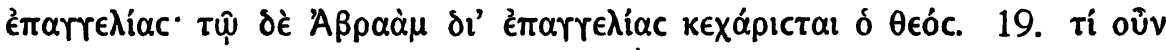

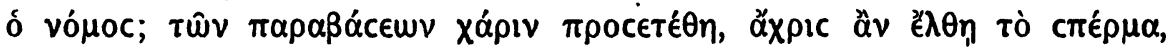

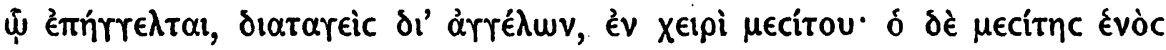
oủx ěctiv, ó dè $\theta$ eòc eỉc éctiv.

Bei seiner Darlegung des Verhältnisses des alttestamentlichen Gesetzes zur Verheißung an Abraham und seinen Samen geht Paulus

* [Es sei die Bemerkung gestattet, daß es sich hier nur um einen Ausschnitt handelt aus einer für November 1914 in Basel geplant gewesenen Rektoratsrede, deren Ausarbeitung die Mobilmachung verhinderte. Eine zeitweilige Dienstleistung hinter der Front infolge einer Erkrankung wurde benutzt, um einen Teil des Themas zu behandeln. Doch war es auch bei dieser Einschränkung nicht möglich, wie beabsichtigt, das ganze Papyrusmaterial und die Inschriften durchzuarbeiten; vielmehr konnten in der Hauptsache nur die in der Chrestomathie von Mitteis und Wilcken zusammengestellten Papyri verarbeitet werden. Auch von einer Prüfung der Verwendung der aus den $\mathrm{Pa}$ pyri der ptolemäischen Zeit bekannten Rechtswörter bei den LXX, dic als Vorarbeit gedacht war, mußte abgesehen werden.]

1 Die Stelle ist bereits von einem Juristen eingehend behandelt: $M$. Conrat, Das Erbrecht im Galaterbrief $(3,15-4,7)$ in dieser Zeitschr. Bd. 5 S. 204 ff. Conrat stellt auf das römische Recht $a b$ und läßt das hellenistische Recht außer Betracht, welches uns namentlich. die auch für den Bearbeiter des römischen Rechts so wichtigen Papyri zeigen. - Von rein juristischen Gesichtspunkten aus behandelt die Stelle auch Halmel, Uber römisches Recht im Galaterbrief, Essen 1895. 
von dem in v. 15 wiedergegebenen Satz menschlichen Rechtes aus. Hierbei drängt sich zunächst die Frage auf; ob sich nachweisen läßt, daß dieser Satz einer bestimmten menschlichen Rechtsordnung entnommen ist - eine Frage, deren Stellung auch für den Theologen nicht müßig ist, da ihre Bejahung unter Umständen neue Gesichtspunkte für das Verständnis der Stelle und auch ein. Steinchen für das Mosaikbild vom Leben und der Arbeit des Apostels Paulus bringt, während es andererseits für den Rechtshistoriker von Interesse ist, zu sehen, welche Rechtsordnung. Paulus „der Nationalität nach Grieche, politisch römischer Untertan, Bürger der dem römischen Reich angehörenden Stadt Tarsus, von Kind auf im Besitz des römischen Bürgerrechts, aus einer Familie mosaischen Glaubens, ohne Zweifel jüdischen Ursprungs " 1 in seinen Briefen, die an die im römischen Reich verstreuten Gemeinden gerichtet sind; berücksichtigt, und von da aus einen Einblick in die komplizierte Rechtswelt der damaligen Zeit zu tun.

Hier im Galaterbrief könnte man daran denken, daß Paulus das Recht der Galater berücksichtigt, ${ }^{2}$ dessen ${ }^{~ F o r t e x i s t e n z ~ n o c h ~ u ̈ b e r ~ d i e ~}$ Zeit des Paulus hinaus durch den römischen Juristen Gaius ${ }^{3}$ aus Anlaß seiner Erörterungen über die römische patria potestas bezeugt ist. Dies scheint aber schon durch die allgemeine Fassung des Satzes (v. 15) ausgeschlossen $\mathrm{zu}$ werden, die darauf hinweist, daß es sich - wenn überhaupt um eine spezielle - um eine in weiten Gebieten maßgebende und allgemein bekannte Rechtsordnung handeln muß.

$\mathrm{Zu}$ denken ist auch an das jüdische Recht, nach welchem die Juden damals lebten. ${ }^{4}$ Doch spricht gegen dessen Verwendung schon der Umstand, daß Paulus von seinen Lesern, die zum großen Teil von Haus aus Nichtjuden waren, ${ }^{5}$ nicht allgemein die Kenntnis dieses

1 Mommsen, Rechtsverhältnisse des Apostels Paulus, in dieser Zeitschrift, Bd. 2 S. 81 ff. (Diese Arbeit kann übrigens auf Grund der aus den Papyri gewonnenen neuen Kenntnisse in manchen Punkten ergänzt werden.)

2 So läßt Sieffert, Das Recht im Neuen Testament, Göttingen 1900 S. $14 \mathrm{ff}$., und in Meyers Kom. z. NT (9. Aufl.) S. 235 die Möglichkeit offen, daß Paulus in Gal. 4, 1.2 auf das Recht der Galater Bezug nimmt; vgl. auch Conrat a. a. O. S. 233 . Siehe dazu aber die Ausführungen zu dieser Stelle unten S. $104 \mathrm{ff}$.

3 Instit. I, 55 nec me praeterit Galatarum gentem credere, in potestate parentum liberos esse. Mitteis, Reichsrecht und Volksrecht in den östlichen Provinzen des römischen Kaiserreichs S. 24.

4 Mitteis, Reichsrecht S. 34.

5 Gal. 4, 8-10. 
Rechts voraussetzen konnte. ${ }^{1}$ Daran ändert auch nichts, wenn sich Paulus an dieser Stelle durch die Art der Beweisführung, die Deu-

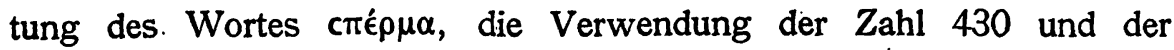
Theorie, daß das Gesetz nicht von Gott direkt, sondern von den Engeln erlassen sei, als Rabbinenschüler erweist. ${ }^{2}$

Es bleiben noch das römische und das hellenistische Recht. Eine weitverbreitete, wenn auch im allgemeinen nur oberflächliche Kenntnis wichtiger Sätze des römischen Privatrechts konnte Paulus wohl auch bei den nicht nach diesem Recht lebenden Provinzialen, ${ }^{3}$ welche in der damaligen Zeit ${ }^{4}$ in der östlichen Hälfte des römischen Kaiserreichs bei weitem die Mehrzahl der Bewohner bildeten, ${ }^{5}$ voraussetzen, namentlich soweit es sich um Rechtssätze handelte; welche die Provinzialen in ihrer Eigenschaft als Nichtbürger berührten. ${ }^{6}$ So ist denn auch von verschiedenen Seiten die Ansicht vertreten worden, daß Paulus in unserer Stelle das römische Recht verwertet. ${ }^{7}$

1 Es erübrigt sich deshalb auch ein Eingehen auf die Geschichte des Testaments im jüdischen Recht, dem es von Haus aus fremd war, siehe Mitteis, Reichsrecht S. 340, Bruck, Schenkung auf den Todesfall im griechischen und römischen Recht (1909) S. 71.

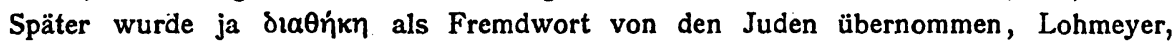
Diatheke S. $29 \mathrm{ff}$. Vgl. über das talmudische Recht Rapaport, Zeitschr. f. vergl. Rechtsw. XV 33. 44. 118.

2 Siehe Deißmann, Paulus S. 72-74.

3 Was speziell das Testament anlangt, so testierte der Provinziale, welcher nicht das römische Bürgerrecht besa $B$, nach seinem Volksrecht, welches auch in den übrigen Fragen des Erbrechts für ihn galt, siehe Mitteis, Reichsrecht S. $102 \mathrm{ff}$. - Dagegen war für den römischen Bürger Paulus hinsichtlich seines eignen Testaments römisches Recht maßgebend, vgl. auch Mitteis, Grundzüge der Papyruskunde (II, 1) S. XVI, 246.

4 Lange vor der Constitutio Antonina, welche im Jahr 212 in weitem Umfang den Provinzialen das römische Bürgerrecht verlieh. Ihr Text ist, wenn auch leider verstümmelt, auf Papyrus bekannt geworden, siehe Mitteis, Chrestomathie der Papyruskunde (II, 2) 377 , Grundzüge S. 288-89.

5 Uber die Verbreitung des römischen Rechts in der östlichen Reichshälfte im ersten Jahrhundert nach Christus vigl. Mitteis, Reichsrecht, namentlich S. 111-153; über die Verhältnisse in Ägypten siehe jetzt Mitteis, Grundzüge, insbesondere S. XVI, XVII.

6 Siehe dazu unten S. 100 A. 2.

7 So von Conrat und Halmel (oben S. 84 Anm. 1), Zahn im Kom. z. NT (Galaterbrief, 1905) S. 163, Sieffert, Das Recht im NT S. $14 \mathrm{ff}$. - Namentlich von Halmel wird die Verbreitung der Kenntnis römischer Rechtssätze in der damaligen Zeit sehr überschätzt. Geht er doch z. B. (S. 7 ff.) so weit, anzunehmen, daß Paulus mit v. 16 den

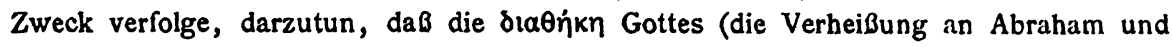
sein $c \pi \epsilon \rho \mu \alpha)$ nach römischem Recht gültig sei, da nach diesem Wortlaut eine konkrete, nach individuellen Merkmalen vorstellbare Person (ctép $\mu \alpha=$ Christus) eingeset/zt sei, während eine Einsetzung von c $\pi \dot{\epsilon} \mu \alpha \tau \alpha$ (=das ganze Volk Israel) nach römischem Recht ungültig gewesen wäre, weil es sich in diesem Fall um personac incertae gehandelt 
Zieht man jedoch auch die hellenistischen Urkunden, speziell

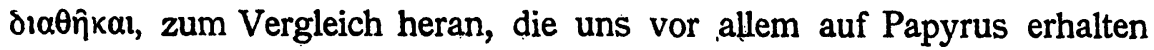
sind, so ergeben sich überwiegende Gründe dafür, daß Paulus bei der Abfassung von v. 15 an derartige $\delta\llcorner\alpha \theta \hat{k} k \alpha$ dachte und damit an das griechische Recht, wie es in der Gestalt des hellenistischen Volksrechts für weite Kreise der Bevölkerung im ganzen Osten des römischen Reiches zur Zeit des Paulus in Geltung war. ${ }^{1}$

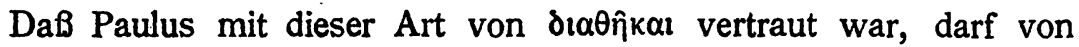
vornherein angenommen werden - er hatte wohl schon früh in seiner Vaterstadt Tarsus ${ }^{2}$ Gelegenheit, davon zu hören und solche Urkunden auch zu sehen; auch konnte er von den Empfängern seines Briefes

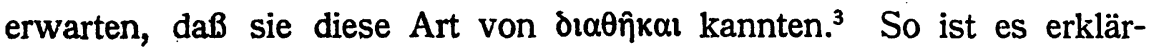
lich, daß Paulus an unserer Stelle technische Ausdrücke der hellenistisc̀hen Rechtssprache verwendet, wie folgende Belege aus den hellenistischen Papyrusurkunden aus Ägypten beweisen. ${ }^{4.5}$

hätte. Danach müßte doch wohl Paulus von den Galatern voraussetzen, daß sie den Satz des römischen Rechts, wonach den personae incertae die testamenti factio passiva fehlt, kennen und ohne weiteres seine dunkle Anspielung auf denselben verstehen würden. Das ist kaum glaublich. - Verfehlt ist z. B. auch, wenn Halmel glaubt, daB

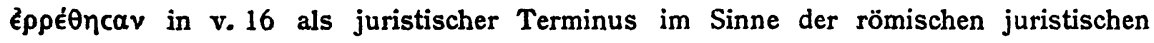
Quellen gebraucht sei; vgl. auch Gen 15,13 unten S. $98^{\circ}$. - V. 16 hat überhaupt keinen spezifisch juristischen Inhalt und ist deshalb für unsere Untersuchung ohne Interesse. Es soll wohl damit dargelegt werden, was unter der $\delta\llcorner a \theta \dot{\eta} k \eta$ Gottes, die mit der menschlichen verglichen werden soll, $\mathrm{zu}$ verstehen ist, und dargetan werden, daß die VerbeiBung sich nicht auf alle physischen Nachkommen Abrahams (= das Volk Israels), sondern nur auf Christus (und durch ihn auf die Christen v. 28/29) bezieht.

1 Mitteis, Reichsrecht, insbes. S. 61; Partsch, Griechisches Bürgschaftsrecht S. 5, Arch. f. Pap. Forsch. VI S. 35. - Allerdings muB bei der Fortentwicklung des hellenistischen Rechts auch mit einer Beeinflussung durch das römische Recht gerechnet werden; eine solche liegt vielleicht vor, wenn in den hellenistischen $\delta \alpha \theta \hat{\eta}$ kal in rö-

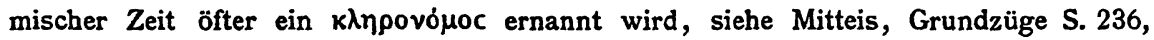
unten S. 97f.; vgl. auch unten S. 107 A 3 (über die vielleicht stattgehabte Nachahmung der römischen cura bei Peregrinen).

2 S. Deißmann, Paulus S. 63.

3 Auch ohne Heranziehung der südgalatischen Hypothese, worach sie in der römischen Provinz Galatien, und zwar in deren südlichem, hellenisiertem Teil zu suchen wären, ist bei den Empfängern des Briefs schon aus dem Umstand, daß Paulus griechisch an sie schreibt, auf Kenntnis der griechischen Sprache und einen gewissen Grad hellenistischer Bildung, damit wohl auch auf ihre Kenntnis hellenistischer Urkunden zu schließen.

4 Wenn sich der juristische Wortschatz des Paulus mit dem der Papyri nicht vollkommen deckt (so ist das Emı Wissens nicht belegt), so kann dịes - abgesehen von der zufälligen Lückenhaftigkeit des Papyrusmaterials - seinen Grund darin haben, daß Paulus nicht sklavisch dem 
Das. Verb kupoûv begegnet als Rechtswort in den Papyri; ${ }^{1}$ doch

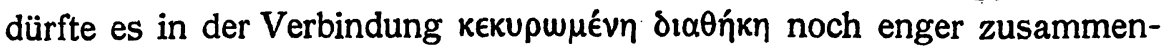

Text der ihm vorschwebenden Urkunden folgt. Andererseits muß man ja im Auge behalten, daß auch mit - für die gegenwärtige Untersuchung nicht wesentlichen Verschiedenheiten in den technischen Ausdrücken der Rechtssprache und der Stilisierung der Papyrusurkunden aus Ägypten gegenüber Urkunden aus anderen Gebieten des hellenistischen Rechtskreises gerechnet werden muß. Außerägyptische, speziell kleinasiatische Urkunden, die hellenistisches Recht widerspiegeln, sind uns ja nicht in der gleichen Menge bekannt. Nur wenige unter den in Ägypten gefundenen griechisch geschriebenen Urkunden zeigen außerägyptische Stilisierung. Einige stammen aus Kleinasien, siehe Wilcken, Arch. f. Papf. II 138, IV 536. Die durch sie beurkundeten Rechtsgeschäfte sind Sklavenkäufe, siehe Mitteis, Grundz. S. 193, Kreller, Erbrechtliche Untersuchungen auf Grund der graeco-aeg. Pap.-Urk. (Leipżiger Diss. 1915) S. 57. Teile eines außerägyptischen Testaments enthält wohl auch B. G. U. 895.

5 Auf die auch sonst begegnende Verwendung zahlreicher hellenistischer Rechtswörter durch Paulus hat zuerst Deißmann hingewiesen; vgl. z. B. die Zusammenstellung im Licht v. O. S. $253^{1}$. - Hier mögen beispielshalber zum Philemonbrief einige Belege genannt werden, die gerade zur Hand sind. Hier ist die Stelle v. 18-20

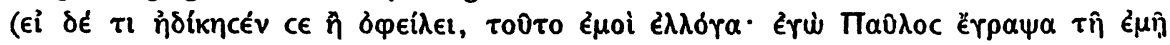

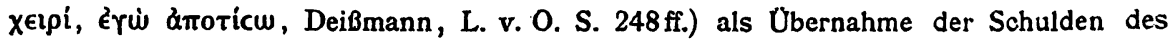

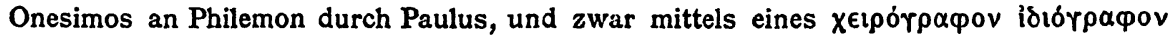
(vgl. z. B. Mitteis, Chrest. 227 1. 14, Mitteis, Grundz. 56; auch P. Oxy 495 Z. 15 8c $\alpha$

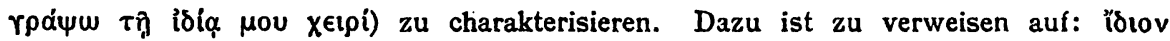

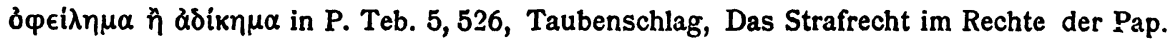

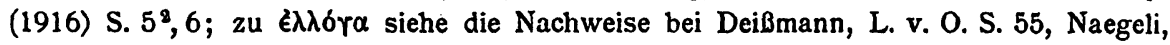
Wortschatz, S. 48 und B.G. U. 1028 (eine Abrechnung, in welcher das Wort öfter begegnet) und vor allem jetzt P. Ryland 243 (II. Jahrh. p. C.): $\delta c \alpha-\epsilon \dot{\alpha} v \alpha \dot{v} \alpha \lambda \dot{\omega} c n c-$

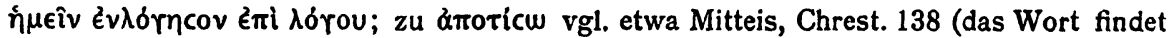
sich regelmäßig in den Strafklauseln, siehe Berger a. a. O. S. 16f.). In folgenden

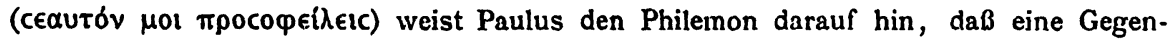

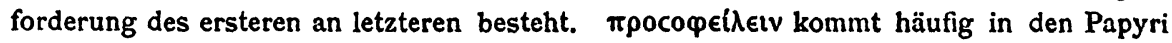
vor und bezeichnet die rückständige Schuld im allgemeinen, z. B. P. Oxy 499 Z. 28 (121. n. C.) $\delta \delta^{\prime} \not \alpha v \pi \rho \circ \alpha^{2}$

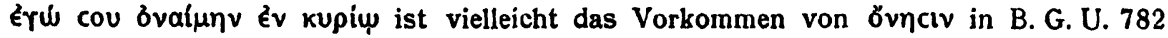
(182/3 n. C.) nicht ohne Interesse. Dieses leider stark zerstörte Stück enthält zunächst das Anerkenntnis einer Darlehensschuld. Dem Gläubiger steht anscheinend an Grund-

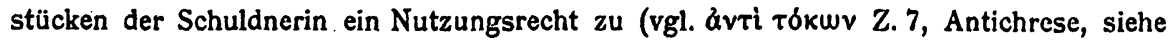
Mitteis, Grundz. S. 152 ff.); in diesem Zusammenhang findet sich hier $6 v \eta\rceil ? v$ (Z. 8

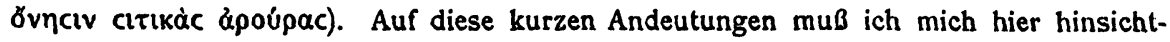
lich des Philemonbriefs beschränken, Näheres an anderem Ort; nur sei bemerkt, daß trotz der juristischen Wendungen - der rechtsgeschäftliche Charakter des Briefs nicht so stark betont werden darf, wie dies Dächsel (Paulus, der Apostel Jesu Christi II. Abt. S. 83 Anm. 21) tut.

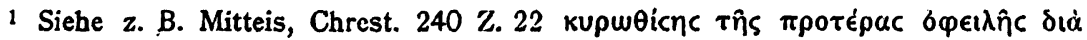
$\tau \hat{\eta} c \mu \in \tau \alpha \gamma \in v \alpha \mu \dot{v}^{\prime} \eta \bar{c}$ (die erste Schuld ist durch den zweiten Schuldschein neu bestätigt; vgl. dazu etwa Mitteis, Chrest. 185 (182/3 n. C.) Z. 20: kupiwv $\mu[\epsilon v o ́ v] \tau \omega v$ 


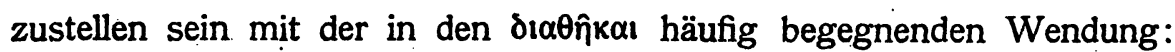

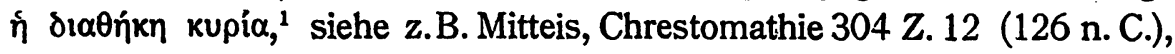
305 Z.30 (156 n. C.), 307 Z. 12 (frühes 2. Jahrh. n. C.). ${ }^{2}$.Man geht wohl

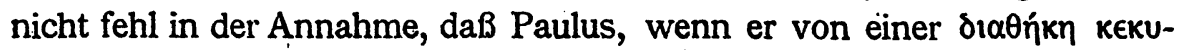

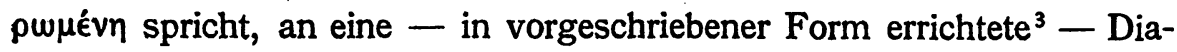
thekenurkunde ${ }^{4}$ denkt, die mit einer solchen Klausel versehen ist, worin der $\delta ı a \theta e ́ \mu \in v o c$ - gegenüber den Zeugen und der Behörde - die Ur-

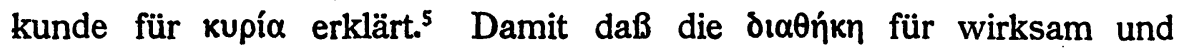
kräftig erklärt wird, ist auch ausgedrückt, daß die Urkunde durch nichts ${ }^{6}$ entkräftet werden, daß nichts kuptú $\tau \in \rho \circ v$ sein soll. ${ }^{7}$ Dieser so gesicherten menschlichen Diathek stellt dann Paulus in v. 17 die

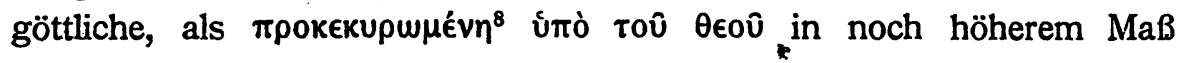

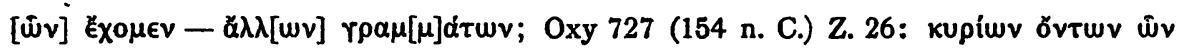

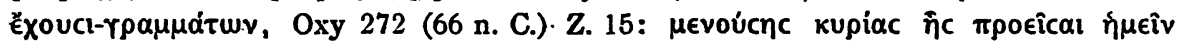

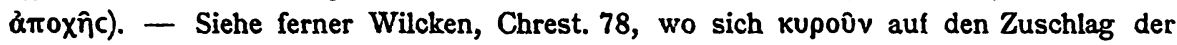
Behörde bei der Versteigerung bezieht, Wilcken, Ostraka I 526; vgl. auch P. Ryland 97, Einleitung. - An die Gottheit wird in einer Orakelfrage (Wilcken, Chrest. 122 vom

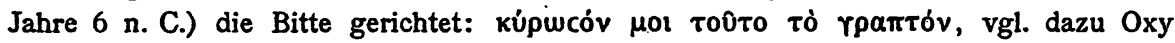

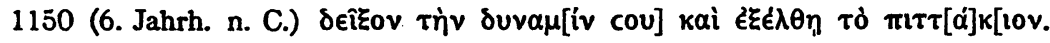

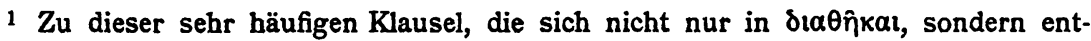
sprechend auch in Urkunden über andere Rechtsgeschäfte findet und die noch näherer Untersuchung bedarf, siehe Mitteis, Reichsrecht S. 178, Partsch, Zeitschr. f. Handelsrecht Bd. 70 S. $447 \mathrm{ff}$., $474 \mathrm{ff}$.

$2 \mathrm{DaB}$ in der ptolemäischen Diathek, Mitteis Chrest. 301 (225 v. C.) die Klausel (die-vor der Nennung der Zeugen zu erwarten wäre) fehlt, hängt vielleicht damit zúsammen, daß statt dessen der König zum Testamentsvollstrecker ernannt ist.

3 In den Papyri sind bis jetzt als Urkundenformen, in welchen die Errichtung

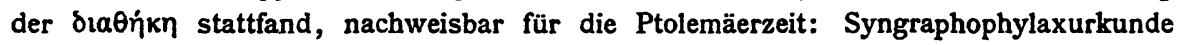

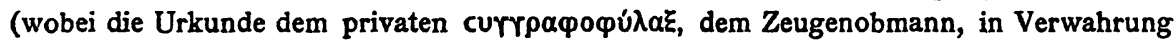
gegeben wird) und vor dem Notar (Agoranomen) errichtete Urkunde. - In römischer Zeit bleibt nur letztere Form, wobei aber auch sechs Zeugen mitwirken. Die Urkunde

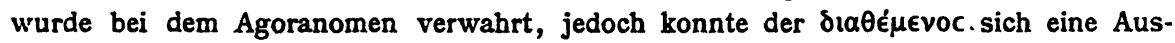
fertigung aushändigen lassen; vgl. Mitteis, Grundzüge S. 236, 53; 62/3.

4 Es kommt wohl nach hellenistischem Recht allgemein das Rechtsgeschäft (das Testament) erst durch Errichtung der (dispositiven) Urkunde zustande.

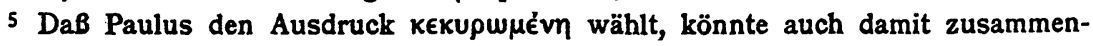

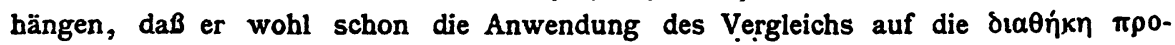

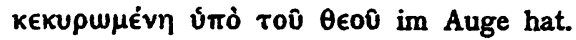

6 Es sei denn durch die Rücknahme seitens des hierzu befugten $\delta \_\theta \in \dot{\mu} \mu \in v o c$ selbst, im Text S. 103.

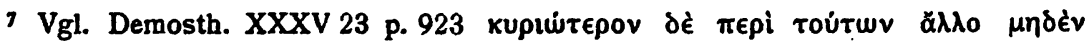

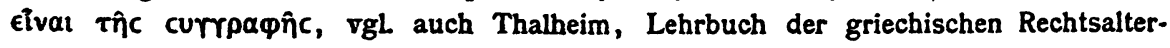
tümer S. 110.

8 Zur Frage, worin hier dieses mpokupoûv besteht, siehe unten S. 101, Anm. 3. 


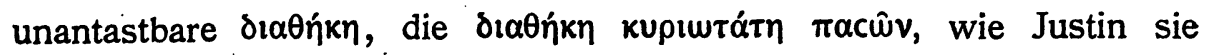
nennt, ${ }^{1}$ gegenüber.

Es muß aber als möglich bezeichnet werden, daß Paulus, wenn

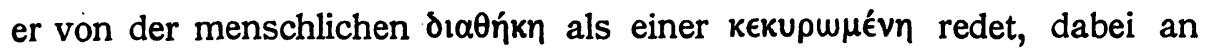
eine solche denkt, die noch durch eine weitere Klausel gesichert ist.

In den drei eben angeführten $\delta 1 \alpha \theta \hat{\eta} \kappa \alpha$ (und auch in anderen) geht

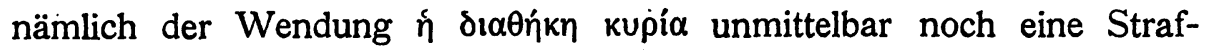
klausel voraus, welche bei Zuwiderhandlungen gegen die Diathek Strafen gegen jeden Zuwiderhandelnden festsetzt. ${ }^{2}$ In 307 lautet dieser

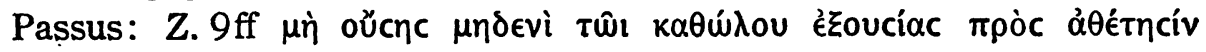

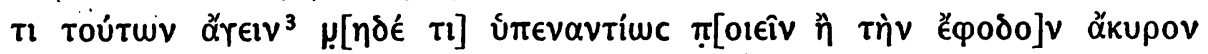

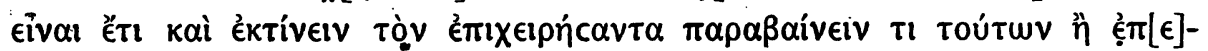

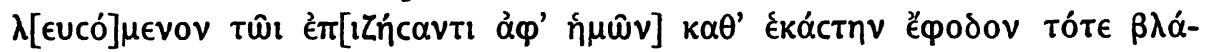

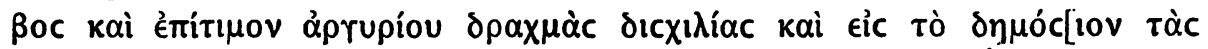

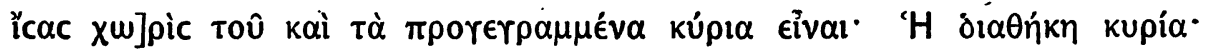
Der Anfang dieser Klausel erinnert sofort an das oủdeic ảt $\epsilon \in \hat{\imath} \hat{\imath}$ in Gal 3, 15 und läßt vermuten, daß Paulus bei der Abfassung von v. 15 an eine solche, ihm aus Strafklauseln bekannte Wendung ${ }^{4}$ sich erinnerte und diese $z \mathrm{u}$ seiner Beweisführung benutzte, worauf später zurückzukommen ist. Vielleicht stellt sich also Paulus unter der $\delta \iota \alpha-$

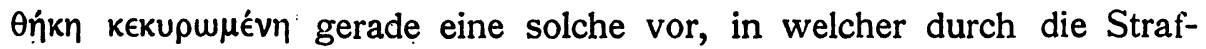
klausel jedem die Macht zum ả $\theta \in \tau \in \hat{v}$ und sonstigen mit der Diathek im Widerspruch stehenden Handlungen abgesprochen und jeder Angriff

1 Vgl. Behm, Der Begriff der $\delta \alpha \theta \dot{\eta} \kappa \eta$ im NT S. 104.

2 Uber diese Strafklauseln, welche mit den weitverbreiteten Sepulkralmulten zusammenzustellen sind, siehe Mitteis, Grundzüge S. 240: „Man wird es auch als sicher annehmen können, daß diese Klauseln rechtlich wirksam waren; das aber kann nur auf gesetzlicher Grundlage der Fall gewesen sein. Also werden gesetzliche Bestimmungen (resp. was den gleichsteht, solche des Provinzialedikts) bestanden haben, welche das Testament unter besonderen Schutz stellten." - Ferner Berger, Strafklauseln in den Papyrusurkunden (1911), insbesondere S. $229 \mathrm{ff}$. über Strafklauseln in letztwilligen Verfügungen; Lohmeyer a. a. O. S. $22^{4}, 26^{2}$.

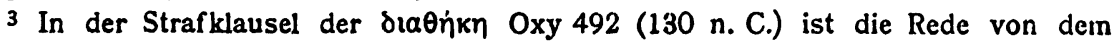

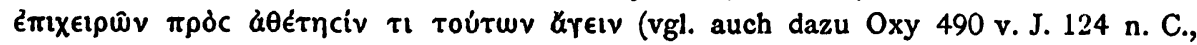

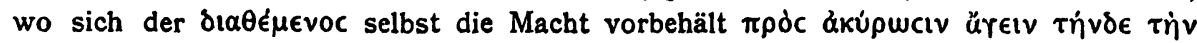

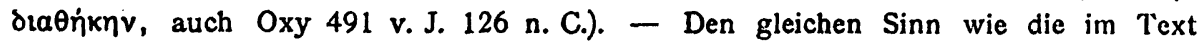
wiedergegebene Formel hat in der im Archiv f. Pap.-Forsch. I S. 64 publizierten dıa

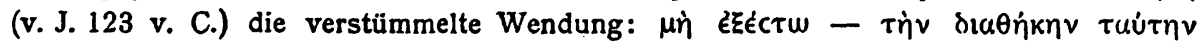

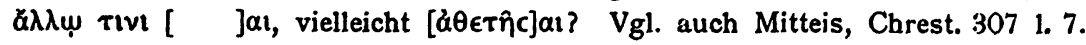

4 Auch in B.G.U. 1123 (aus augusteischer Zeit) heißt es in der Strafklausel:

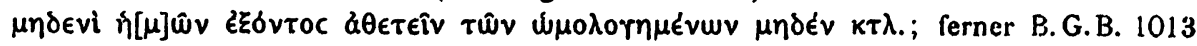

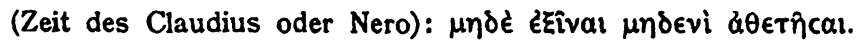


für ắkupoc und strafbar, dann noch durch die folgende Klausel die

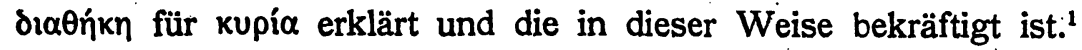

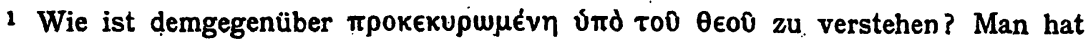
das $\pi \rho 0$ in $\pi \rho \circ \kappa \in K \cup \rho$. als korrelat mit dem folgenden $\mu \in \tau \dot{\alpha}$ aufgefaßt, siehe Sieffert,

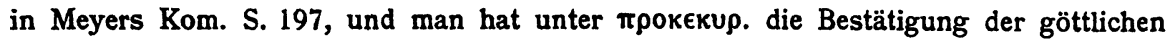

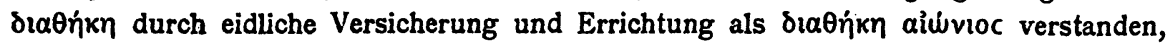
Zahn a. a. O. S. 170; Gen 17, 7; 22, 16-18; Hebr 6, 13-18. Es ist aber wohl auch folgende Erklärung zu erwägen: Dabei ist auszugehen von der Annahme, daß Paulus hier an Gen 15,8ff. denkt (siehe unten S. 98, Anm. 4). Hier heißt es in v. 18: Ékeî

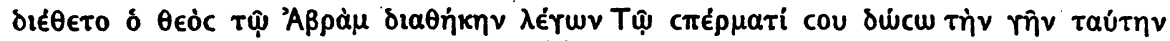
$\kappa \tau \lambda$. Diesem Satz geht voraus die Schilderung eines Vorgangs, durch welchen Gott diese Zusage bekräftigt, v. 17 (s. dazu Lohmeyer a. a. O. S. $42^{1}, 46^{4}$ ). In diesem Vorgang, speziell dem Hindurchgehen des Feuers, könnte Paulus ein kupouvv erblicken. (Dabei ist auch an die oben S. 88, Anm. 1 wiedergegebene Wendung: kúpwcóv $\mu 01$

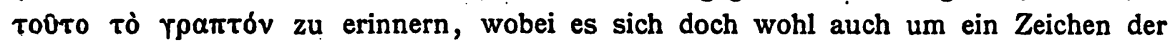
Gottheit handelt).

Bezieht man $\pi \rho \circ$ Kupoûv, wie eben angedeutet, auf den in Gen. 15, 17 geschilderten Vorgang, so könnte auch das $\pi \rho 0$ hier einen anderen Sinn haben, als seither angenommen wurde. Treffen nämlich die oben im Text gemachten Ausführungen zu,

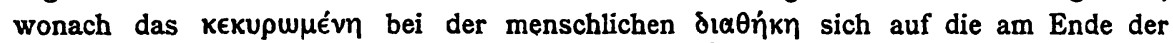
$\delta ı \theta \dot{j} \kappa \eta$ stehende kupía-Klausel (vielleicht in Verbindung mit der Strafklausel) bezieht,

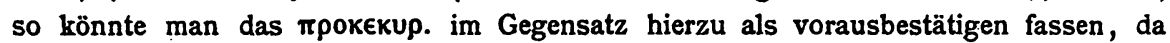
der bekräftigende Vorgang (v. 17) der Errichtung der $\delta\llcorner\alpha \dot{\eta} \kappa \eta$ (v. 18) vorausgeht.

Hält man dagegen an der Beziehung des mpokekup. auf den Schwur Gottes, durch welchen er die $\delta ı \theta \dot{j} \kappa \eta$ bekräftigt, fest, so wird man dadurch auf Testamente hingelenkt, die von dem Testator beschworen und für ihn unwiderruflich sind. Ein solcher Eid des Testators findet sich in dem Testament des Abraham von Hermonthis (Mitteis, Chrest. 319, vom Ende des 6. Jahrh. n. C.), der es selbst als a $\mu \in \tau \alpha \mu \epsilon \hat{\lambda} \eta \tau$ iov bezeichnet (Z. 4) und Z. $59 \mathrm{ff}$. auf Widerruf verzichtet und beschwört. Man könnte dabei versucht sein, zu glauben, daß Paulus, wenn er von der menschlichen $\delta i \alpha \theta$.

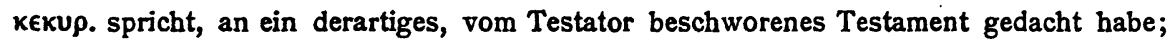
das erscheint jedoch ausgeschlossen, da zu seiner Zeit, soviel wir bis jetzt wissen,

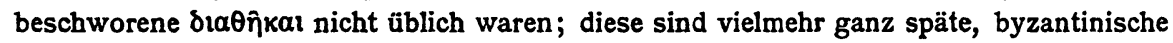
Gebilde; siehe Wenger, Z. Sav. St. XXIII S. 232 ff., 265 ff., XXXII S. 337, Partsch, Ztschr. f. Handelsrecht Bd. 70 S. 442.

Das Wort $\alpha \mu \in \tau \alpha \mu \in \lambda^{\prime} \lambda \tau \tau o v$, das eben im Testament des Abraham v. H. begegnete und das auch z. B. in einer Schenkung auf den Todesfall aus sehr später Zeit (Ztschr. d. Sav. St. XXXII S. $326 \mathrm{ff}$.) wiederkehrt (in früheren Urkunden findet man ả $\mu \in \tau \alpha v o ́ \eta \tau o c$, vgl. Deißmann, Neue Bibelst. S. 84, Nägeli, Wortschatz S. 45 , ferner z. B. P. Flor. 47 [213/7 n. C.], P. Cairo Preis. 42 [3./4. Jahrh. n. C.]), .wird auch von Paulus gebraucht,

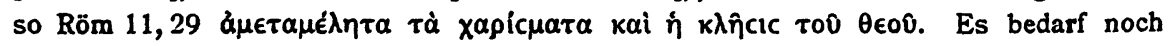
einer näheren Untersuchung am ganzen Material, wie weit sich die Beobachtung bestätigt, daß noch öfter Wörter, die sich im NT finden, in dem Urkundenstil der byzantinischen Zeit erscheinen, während sie in ptolemäischer und römischer (speziell früherer römischer) Zeit als Rechtswörter in den Papyrusurkunden nicht üblich sind; wenn diese Untersuchung positiv ausfallt, wird $z u$ fragen sein, wie sich diese Erscheinung erkjärt. 


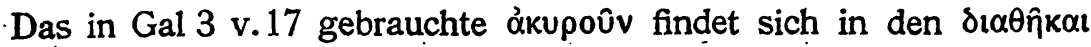
auf Papyrus häufig in einer Klausel, die z.B. in Mitteis; Chrest. 304 Z. 3

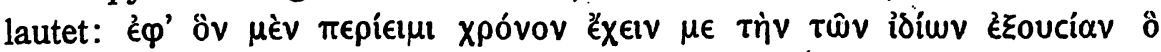

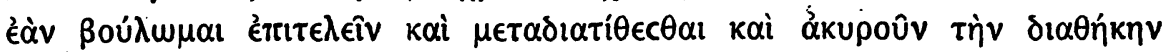

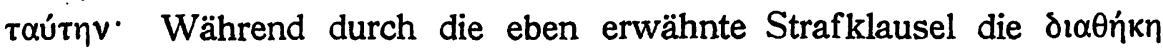
gegen Angriffe von Dritten gesichert werden sollte, behält sich selbst

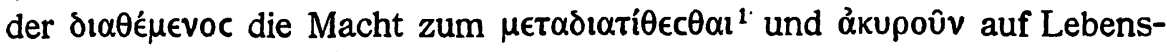
zeit vor. So zeigt uns auch P. Oxy 107 (v. J. 123 n. C.) Z. 7 die Rück-

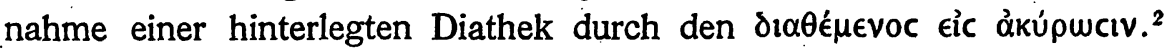

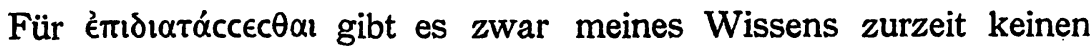
Beleg in den Papyrusdiatheken, wohl aber erscheint hier dıatáccelv als

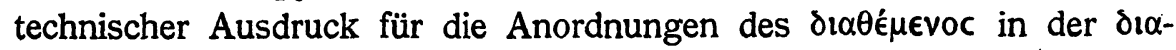

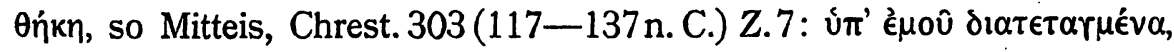

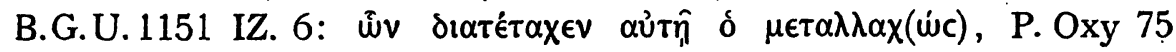
(129 n. C.), Z.30; vgl. auch dı $\alpha \tau \alpha \hat{i} c \alpha-k \alpha \tau \grave{\alpha} \mu[\epsilon \rho ı \tau] \epsilon i \alpha v=z$ ugewiesen in einer elterlichen Teilung ${ }^{3}$ in Mitteis, Chrest. $315 \mathrm{Z} .13$ und dı $\alpha$ táccetv ebenda $307 \mathrm{Z} .3$, wo es sich auf das dem überlebenden Ehegatten zu-

$1 \mathrm{Zu} \alpha \mu \in \tau \dot{\theta} \theta \in \tau o v$ in Hebr. 6,17.18 vgl. Mitteis, Grundzüge S. 241 und z. B.

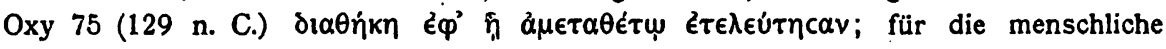
Diathek wird diese Bezeichnung nur für die Zeit nach dem Tode des $\delta \iota \theta \theta \epsilon \in \in v o c$ gebraucht und kann auch nur nach diesem Zeitpunkt gebraucht werden, weil es sich hier um eine letztwillige, $d . h$. bis zum Tod widerrufliche Verfügung handelt.

2 Mitteis, Grundzüge S. 241. - Vgl. dazu die in Quittungen häufige Wendung,

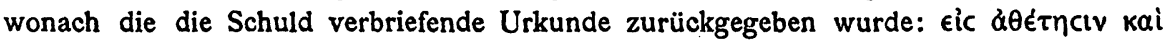
¿kúpwcıv (z. B. Mitteis, Chrest. 163 Z. 21 [v. J. 109 n. C.]). oder nur Gic åkúpwcıv (Mitteis, Chrest. 178 Z. 13 [103 n. C.]) und die Scheidungsakte Mitteis, Chrest. 292 (96 n. C.) und 293 Z. 19-20 (123 n. C.), in welchen gesagt ist, daß der (den Empfang

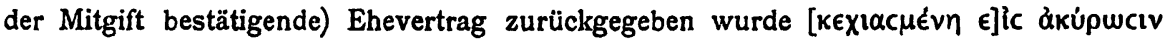

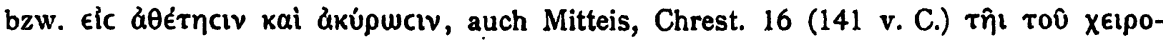

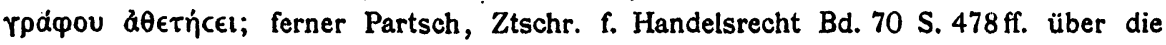
noch weiter zu prüfende Frage, wieweit die beurkundete Schuld bis zur Vernichtung oder juristischen Entkräftung der dispositiven Urkunde weiter besteht. - Für die beim

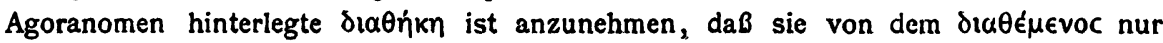
durch Rücknahme (s. oben P. Oxy 107) aufgehoben wurde, also nicht etwa durch Vernichtung der ihm ausgehändigten Ausfertigung. Veränderungen an der letzteren durch den $\delta \_a \theta \in \mu^{\prime} \in v_{0}$ hatten nur Wirkung, wenn er sich ausdrücklich in der Diathek die Befugnis zu Zusätzen vorbehalten hatte, siehe darüber alsbald im Text. Außerdem

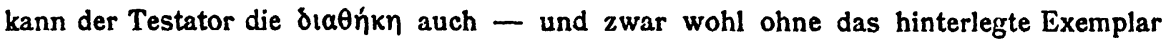
zurückzunehmen - durch eine neue, formgültig errichtete Diathek aufheben (vgl.

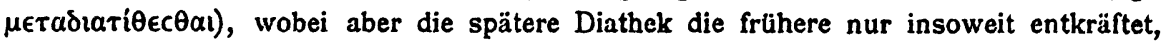
als sie derselben inhaltlich widerspricht, siehe Mitteis, Grundz. S. 241.

3 Vgl. Mitteis, Grundz. S. 244 ff., Rabel, Elterl. Teilung, Festschr. \%. 49. Versammlung deutscher Philologen, Basel 1907; vgl. dazu Lc 12, 13. 14. 
stehende Recht der Verteilung des Nachlasses an die gemeinsamen Kinder bezieht. - In Mitteis, Chrest. 305 (156 n. C.) Z. 25 ff. behält sich

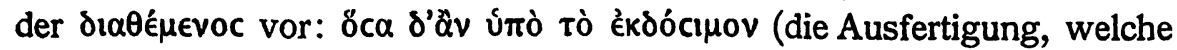
ihm nach Hinterlegung der $\delta\llcorner\alpha \hat{\eta}$ kn beim Agoranomen ausgehändigt

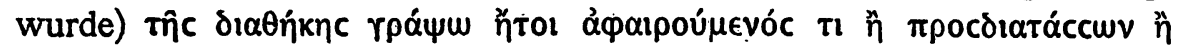

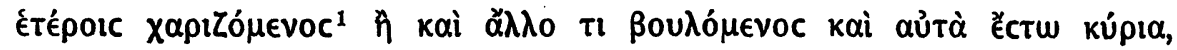
gleich als ob es in der $\delta ı \theta \eta \dot{\eta} \kappa \eta$ niedergeschrieben wäre; wir haben es hier mit einem zum voraus bestätigten Kodizill (codicillus testamenti confirmatus) $\mathrm{zu}$ tun. ${ }^{2}$

Nach den seitherigen Ausführungen, insbesondere angesichts der Tatsache, daß v. 15 fast nur aus der hellenistischen Rechtssprache entnommenen Wörtern besteht, darf man wohl, wie schon erwähnt, annehmen, daß Paulus an eine hellenistische $\delta\llcorner\alpha \dot{\eta}$ kn denkt. Und es ist weiter zu vermuten, daß er speziell durch die Erinnerung an eine Klausel derselben beeinflußt ist, die in ihrem Charakter übereinstimmt

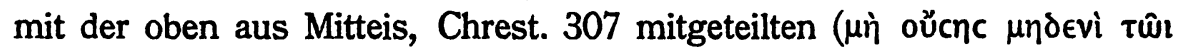

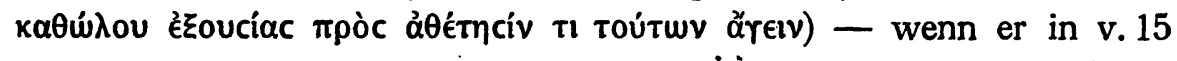

1 Siehe dazu kexápictai in Gal 3, 18.

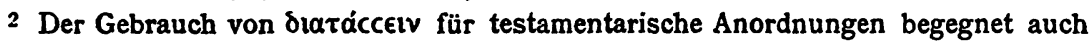
in den kleinasiatischen Inschriften, siehe W. Iudeich im IV. Ergänzungsheft d. Jahrb.

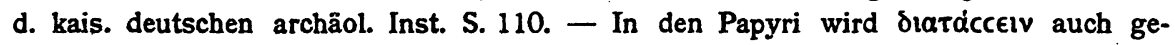
sagt für Anordnungen von Behörden; vgl. Wilcken, Chrest. 361 (200 n. C.), wo es sich um Zuweisung von Grundstücken durch behördliche Anordnung im Wege des

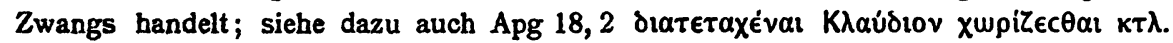
und auch die militärischen Befehle Apg 23, 31 ; 24, 23; in diesem Zusammenhang dürfte auch die Verwendung des Wortes diatáccw durch Paulus bei seinen Anordnungen zur Regelung der Verhältnisse in den Gemeinden zu beachten sein: 1. Kor 7, 17; 11, 34; 16, 1 (Tit 1, 5). - In Chrest. 361 erscheint (Z. 43 und 47) als Substantiv $\mu \in \tau \alpha \delta 1 \alpha$ -

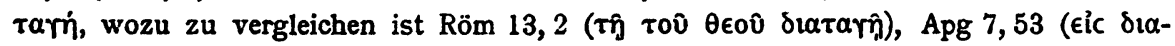

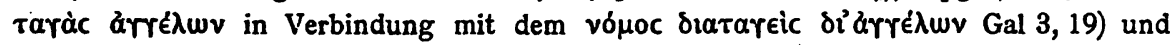

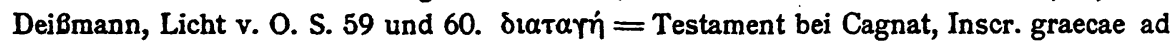
res rom. pert. VI $661^{12}$ und $840^{\circ}$. - In den Papyri (und in Inschriften) erscheint

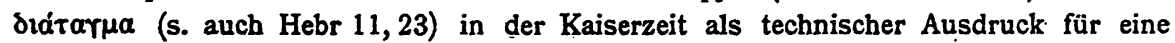
besondere Art amtlicher Erlasse, die Edikte (des Kaisers und der Beamten), vgl. P. M. Meyer, P. Giss. II S. 26; ein solches Edikt ist z. B. die Constitutio Antonina

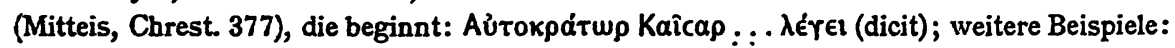
Wilcken, Chrest. 373,439; vgl. auch Deißmann, Licht v. O. S. 285 zu $\tau \delta \delta \epsilon ~ \lambda \epsilon ́ r \in \mathrm{L}$

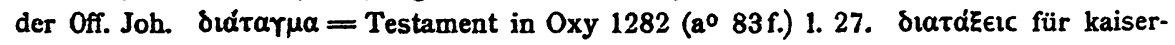

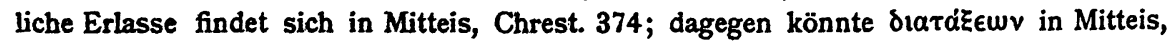
Chrest. 310 (Gesuch um Testamentseröffnung) auf die in der $\delta 1 \alpha \theta \dot{\eta} k \eta$ enthaltenen Anordnungen des Erblassers zu beziehen sein, namentlich wenn die vón Iudeich a. a. $O$.

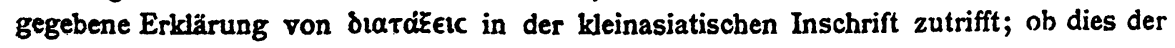
Fall ist, erscheint jedoch sehr zweifelhaft. 


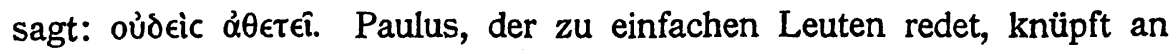
das sie umgebende, lebendige Recht, an einen Satz aus einem verbreiteten Diathekenformular an, wobei es durchaus verständlich wird, wie er gerade auf diesen Satz kommt und ihn in v. 15 als einen allgemein bekannten hinstellt. Dabei kommt noch bestärkend in Betracht, daß Paulus bei dem kurz vorhergehenden "freien" Zitat aus den LXX

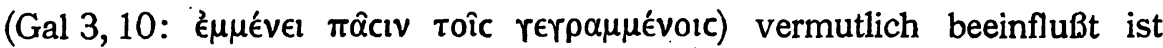
durch eine auch in Urkunden auf Papyrus häufige Wendung, die sich in diesen auch gerade im Zusammenhang mit der Strafklausel findet. ${ }^{1}$ Und weiter spricht für diese Auffassung der Umstand, daß auch als-

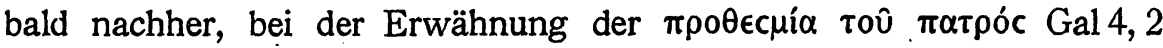
Paulus (wie.später darzulegen ist) ${ }^{2}$ an Bestimmungen dachte, die in den hellenistischen $\delta \iota \theta \hat{\eta} \kappa \alpha \mathfrak{l}$ der damaligen Zeit begegnen.

Ist das oủdeíc in Anlehnung an die erwähnte Klausel des Dia-

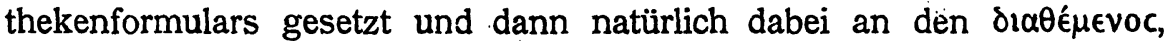
der das Wort in der Urkunde gebraucht, gar nicht gedacht, ${ }^{3}$ so entfallen große Schwierigkeiten, die sich seither manchen Auslegern aus dieser Fassung des Satzes ergeben haben. Hat man doch mitunter

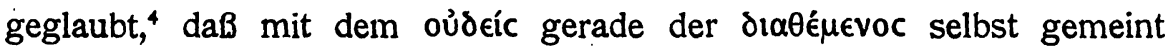
sei, wobei man an ein auch für ihn unantastbares, unwiderrufliches ${ }^{5}$ Adoptionstestament ${ }^{6}$ dachte. $\mathrm{Zu}$ diesem letzteren Punkt ist $\mathrm{zu}$ bemerken, daß zur Zeit des Paulus im hellenistischen Rechtskreis das Adoptionstestament anscheinend schon lange von einer anderen Testamentsart verdrängt worden war, wobei keine Adoption stattfand. ${ }^{7}$ Paulus

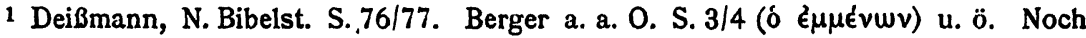
in dem sehr späten Testament des Abraham von Hermonthis heißt es nach der Straf-

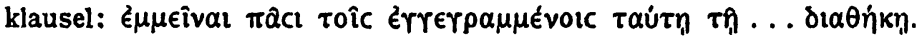

2 Siehe unten S. $107 \mathrm{f}$.

3 Zudem behält ja der $\delta\llcorner\alpha \theta \in ́ \mu \in v o c$ sich selbst regelmäßig ausdrücklich die Macht zum ákupoôv und $\mu \in \tau a \delta i a \tau i \theta \in c \theta a l$ vor, wie oben ausgeführt wurde.

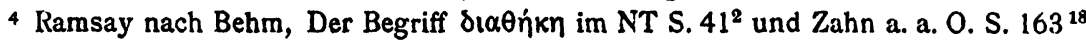
und $164^{20}$.

5 Auf die Frage der Widerruflichkeit des Adoptionstestaments durch den adoptierenden Testator ist hier - da an ein solches nicht zu denken ist - nicht weiter einzugehen; vgl. aber für dieselbe Schulin, Das griech. Testament verglichen mit d. röm. Rektoratsprogr., Basel 1882; Rabel, Z. Sav. St. XXX 470.

6 Auch Deißmann, Paulus S. 102/3, denkt an Adoptionstestament. Die von inm angezogene, ebengenannte Schrift von Schulin behandelt das ältere griechische Recht. Die Frage, ob das Adoptionstestament durch das Testament ohne Elcroíncic mit der Zeit ganz verdrängt wurde, läßt Schulin a. a. 0. S. 33 für das attische Recht offen.

7 Bruck, Schenkung S. 97, 150. - Meines Wissens ist aus der Zeit des Paulus und auch aus erheblich früherer Zeit kein Beleg für testamentarische Adoption aus dem helle- 
hat wohl im v. 15 diese letztere, zu seiner Zeit übliche, hellenistische

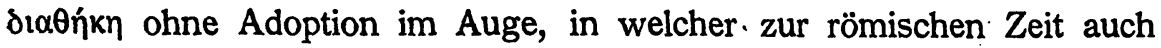

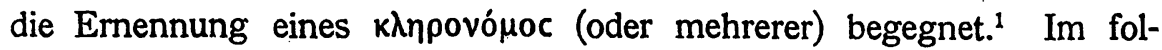
genden verwendet zwar Paulus auch das Bild der Adoption (vio $\theta \in c i \alpha$ ), ${ }^{2}$ die ebenfalls für die Adoptierten als vioi $\theta \in 00$ ein Erbrecht begründet; aber dabei ist nicht an testamentarische Adoption. gedacht. Vielmehr

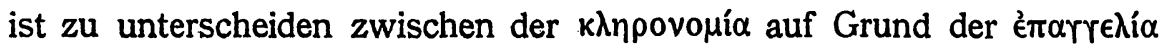

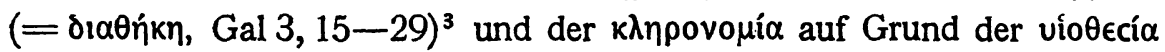
(Gal 4, 4ff.); ${ }^{4}$ diese Unterscheidung zeigt sich evident im Römerbrief. Hier werden, schon räumlich getrennt, deutlich auseinandergehalten:

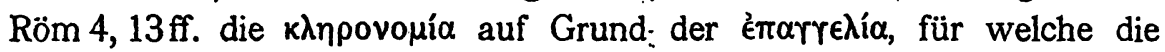
mícrıc Voraussetzung ist - Röm 8,14ff. die $k \lambda \eta p o v o \mu i \alpha$ auf Grund der durch das $\pi v \in \hat{v} \mu \alpha$ vermittelten vio $\theta \in \epsilon^{\prime} \alpha^{5}{ }^{5}$ Paulus häuft auch hier die

nistischen Rechtsgebiet bekannt. Die von Lohmeyer a. a. O. S. $17^{2}$ zitierte Inschrift (J. G. XII, 3. Suppl. Nr. 1402, 1403 cf. M. D. J. A. 25 [1900] 464) ist nicht beweiskräftig,

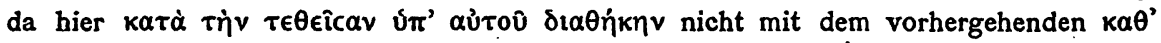

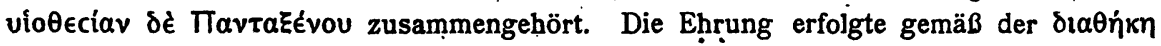

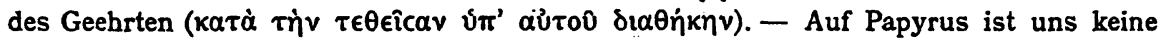
testamentarische Adoption erhalten. Wohl aber wird bei einer Adoption unter Lebenden (Mitteis, Chrest. 363) in der Urkunde gesagt, daß das adoptierte Kind, das nach Z. 15/16

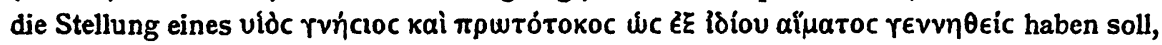

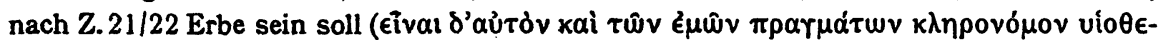

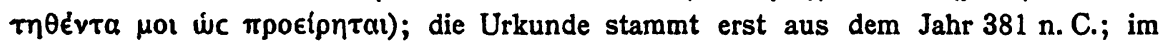
übrigen sind die Ausführungen von Mitteis, Grundz. S. 274/5 zu vergleichen. - Einen weiteren Adoptionsvertrag unter Lebenden enthält. P. Oxy 1206 (335 n. C.).

1 Mitteis, Grundz. 236.

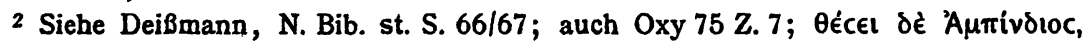
502 Z. 7; 504 Z. $4 . \quad$ Gegensatz: 甲úcel víoc Oxy 1266 Z. 33.

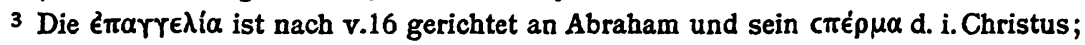

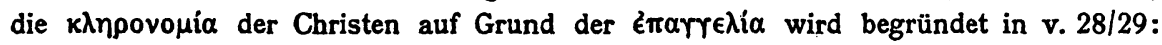

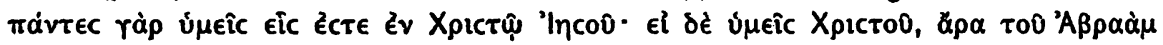

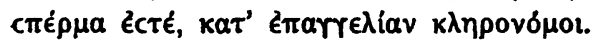

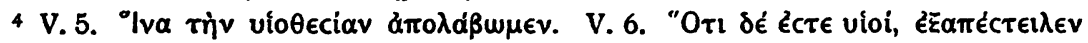

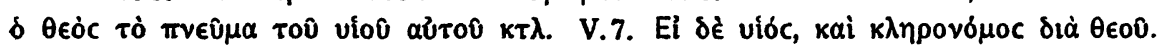

5 Paulus denkt, wenn er von vio $\theta \in c i \alpha$ redet, zum Vergleich an menschliche Verhältnisse, die sich in seiner Umgebung ihm darboten und die wohl überwiegend nach bellenistischem Recht $z u$ beurteilen waren. - Nach älterem griechischen Recht hatte schon der unter Lebenden Adoptierte ein Intestaterbrecht gegen den Adoptierenden, Schulin a.a. O. S. 20; Bruck, Schenkung S. 11, 94. Dieses Erbrecht besteht nach attischem Recht auch neben dem leiblichen Sohn, siehe Meier-Schömann-Lipsius, Der attische ProzeB

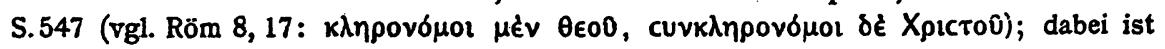
allerdings $\mathrm{zu}$ beachten, $\mathrm{daB}$ es sich im attischen Recht nur um nach der Adoption geborene, leibliche Söhne des Adoptierenden handelt, da beim Vorhandensein von solchen eine Adoption regelmäBig ausgeschlossen war, Schulin a. a. O. 19. Dagegen war z. B. 
Bilder, wie ja auch in Gal 3,13 und 4, 14 das Bild des Freikaufs hineinverwoben ist.

Die Beziehung von oủdeic auf den $\delta \prec \alpha \theta e ́ \mu \in v o c$ ist also abzulehnen.

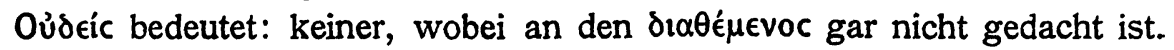
Jeder Zweifel in dieser Hinsicht wäre ja ausgeschlossen, wenn sich dartun ließe, daß Paulus an die $\delta\llcorner\theta \eta \hat{k} k \eta$ eines bereits gestorbenen Menschen denkt; aber wenn auch eine große Wahrscheinlichkeit hierfür zu

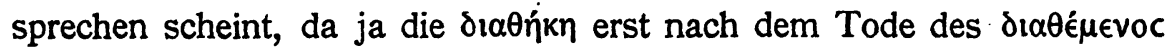
(und ihrer Eröffnung) ${ }^{1}$ ihre Wirkung äußert und auch die Strafklausel, die Paulus vermutlich beeinflußt, sich auf Verstöße gegen die Diathek nach dem Tode des $\delta \imath^{\prime} \theta_{\text {é } \mu \in v o c}$ bezieht, so läßt sich doch aus dem Wortlaut von v. 15 hierauf kein sicherer Schluß ziehen. ${ }^{2}$

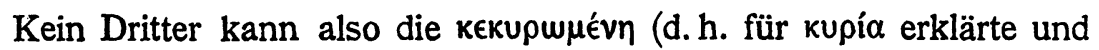
wohl auch durch Strafklausel gesicherte) $\delta$ ianjkn eines Menschen ent-

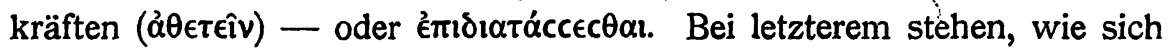

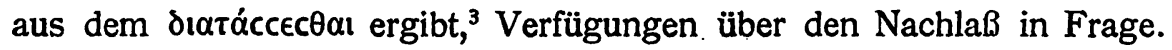
Ein Dritter kann nicht über den Nachlaß verfügen, bezüglich dessen

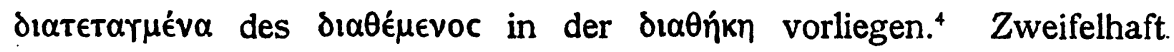

in Gortyn die Adoption zulässig, auch wenn leibliche Söhne vorhanden waren, Bruck a. a. O. S. 11. - Darüber, daß bei der viöecía mosaisches Recht nicht in Frage kommt, siehe Sieffert, Das Recht im NT S. 14 ff., doch denkt S. selbst an römisches Recht.

1 Mitteis, Grundz. 241.

2 Halmel (a. a. O. S. 6) nimmt an, daß der Testator noch am Leben ist, und gibt oủঠєic $k \tau \lambda$. wieder mit: keiner kann ein Testament umstoßen oder hinzuverordnen - der Urbeber natürlich ausgenommen. Er glaubt denn auch, daß P. gerade daran denkt, $\mathrm{daB}$ der Testator bei seinen Lebzeiten ein Kodizill hinzufügt, wobei H. dann auch den vó $\mathrm{oc}_{\mathrm{c}}$ als Kodizill des Testators (Gottes) ansieht. Wenn aber mit v. 5 hätte gesagt werden sollen: der Testator - kein anderer - kann umstoßen oder Zusätze machen, dann wäre dieser wichtigste Punkt (die Befugnis des Testators) gar nicht angedeutet, und nur durch komplizierte Denkoperationen könnte man ihn herauslesen. P. hätte, wenn v. 15 den von $H$. gewollten Sinn hätte haben sollen, sich sicher anders ausgedrückt; statt nur unvollständig negativ, hätte $P$. wohl positiv gesagt: der Testator kann einem Testament Zusätze machen, ohne es kraftlos zu machen. - Wie P. zur Fassung von v. $15 \mathrm{kam}$, wurde oben darzutun versucht.

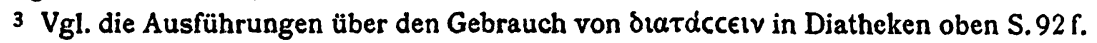

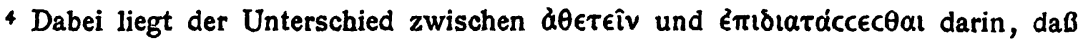
bei ersterem nur die Beseitigung der $\delta a \theta \eta n k n$ in Frage kommt, ohne daß andere, private Bestimmungen an die Stelle der in der $\delta a \theta \dot{\eta} k \eta$ enthalten gewesenen treten, so daß

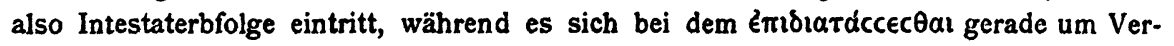
fügungen Dritter über den Nachlal handelt, die zu denen der Diathek hinzutreten oder auch - soweit der Inhalt der neuen Verfügung dem der früheren widerspricht - crsetzen sollen. 
bleibt dabei, in welcher Form sich Paulus dieses émidıatóccec $\theta \alpha \imath$ vorstellt, eine Unklarheit, die damit zusammenhängt, daß die Bedeutung des $E \pi \imath$ hier nicht sicher zu ermitteln ist. Möglich ist die Annahme,

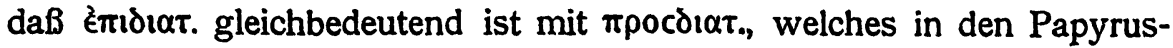
diatheken vorkommt. ${ }^{1}$ Dort macht der $\delta \prec \alpha \theta \in \in \mu \in v o c$, wie schon ausgeführt, ${ }^{2}$ den Vorbehalt, daß alles, was er auf die (ihm nach der Hinter-

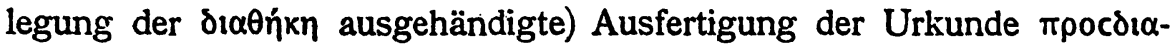

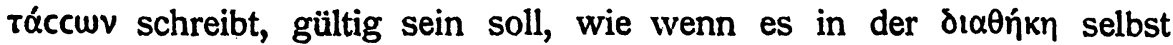

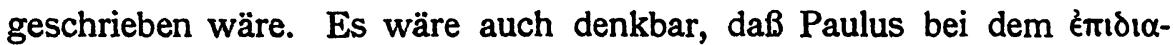
$\tau \alpha \dot{c c c} \in c \theta \alpha$ hinsichtlich der menschlichen $\delta \iota \alpha \theta \dot{\eta} \kappa \eta$ gleichfalls an Zusätze auf der Urkunde durch einen Dritten denkt, die keine Geltung haben - im Gegensatz zu derartigen Zusätzen des $\delta\llcorner\alpha \theta$ é $\mu \in v o c$, der einen dahingehenden Vorbehalt gemacht hat. ${ }^{3}$ Es würde sich also bei dem

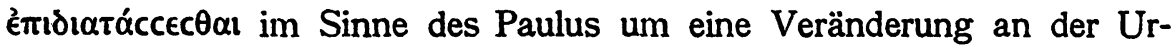
kunde handeln. Eine dahingehende Bestimmung könnte wohl auch in einer Strafklausel enthalten gewesen sein, in Anschluß an welche Paulus vermutlich oủdєic ả $\theta \in \tau \in \hat{\imath}$. in v. 15 gesetzt hat. ${ }^{*}$ Jedoch muß es

1 Siehe auch Meyers Kom. z. St. S. 192; Conrat a. a. O. S. 214.

2 Oben S. 93.

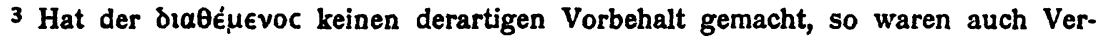
änderungen, die er selbst mit der in seinem Besitz befindlichen Ausfertigung vornahm, wirkungslos (s. auch oben S. 92 Anm. 2). Er behielt aber doch immer noch auf Grund des zu Beginn der Urkunde gemachten Vorbehalts das Recht zum ákupoov und $\mu \in \tau a \delta$ i$\tau\{\theta \in c \theta \alpha$, also zum Widerruf der ganzen Diathek (durch Rücknahme vom Agoranomen) und zur Errichtung einer neuen Diathek. In Mitteis, Chrest. 305 finden sich beide Vorbehalte nebeneinander Z. 4 u. 25.

4 Allerdings richten sich die bis jetzt in den Papyri bekannt gewordenen Strafklauseln im allgemeinen nicht gegen Veränderungen, die an der Urkunde vorgenommen werden könnten, sondern sie beziehen sich auf die Sicherung der Durchführung des beurkundeten Geschäfts. Immerhin ist wohl gerade bei den Wendungen: $\pi \rho \dot{d} c \dot{\alpha} \theta \dot{\epsilon} \tau \eta \dot{ }-$ civ ăreıv u. ähnl. (oben S. 6) auch mit an Außerkraftsetzen der Urkunde durch Vernichtung oder Durchstreichung zu denken. - Die bei Deißmann, Bibelstudien S. 110 mitgeteilte Stelle aus Ep. Arist. (ed. M. Schmidt S. 68, 1 ff.) bezieht sich auf eine Klausel,

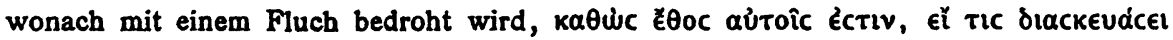

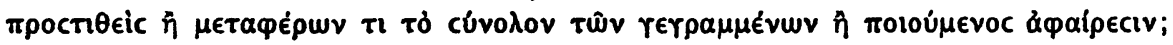
sie wendet sich also nur gegen Veränderungen der Urkunde. Hierfür gibt es meines Wissens in den Papyri keinen Beleg. - Wenn bei der Einreichung von Urkunden bei der Behörde zur Einregistrierung geprüft wird, ob dieselben „frei von Rasur und Zusätzen " sind, und wenn dementsprechend in der Urkunde gesagt wird, dab sie sei:

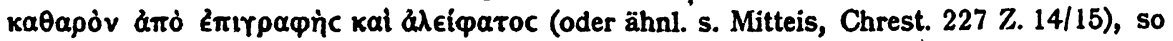
kommt dabei nicht nur Verfälschung der Urkunde durch Dritte in Frage, vielmehr können die Mängel auch von dem Aussteller selbst herrühren, vgl. Mitteis, Chrest. 188 Col. I Z. 12 ff., Wilcken, Archiv f. Pap.-Forsch. I S. 125.

Zeitschr. f. d. neutest. Wiss. 18. Jahrg. 1917. 


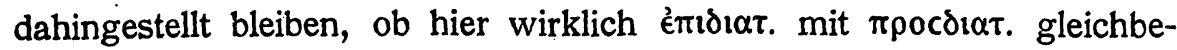
deutend ist. ${ }^{1}$

Unter der menschlichen $\delta เ \alpha \theta \dot{\eta} \kappa \eta$, von welcher das Ebengesagte nach v. 15 gilt, versteht Paulus, wie schon erwähnt, die zu seiner Zeit weitverbreitete hellenistische $\delta \_\alpha \hat{\eta} \kappa \eta$, die sich als Rechtsgeschäft von Todeswegen darstellt, worin der $\delta \alpha \theta^{\prime} \in \operatorname{\mu \in v}$ oc über sein Vermögen (über das ganze oder über Teile desselben) zugunsten anderer verfügt, welche dies Vermögen nach seinem Tode im Wege der Erbfolge erlangen

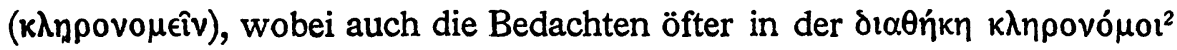
genannt werden.

Nun bezeichnet aber Paulus im v. 17 auch die göttliche Verheißung ${ }^{3}$ an Abraham und seinen Samen im Anschluß an die LXX ${ }^{4}$ als $\delta ı \theta \eta \dot{\eta} k \eta^{5}$

$1 \mathrm{Zu}$ beachten ist demgegenüber auch die Verwendung von $\epsilon \pi \mathrm{t}$ - in feindlichem

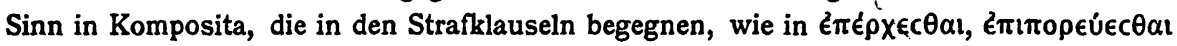
(Berger a. a. O. S. 126).

2 Mitteis, Grundzüge S. 236. 239. - Vgl. dazu Kreller a. a. O. S. 58, wonach in

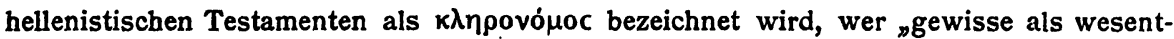

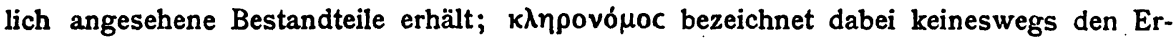
werber des ganzen Nachlasses oder einer Nachlaßquote, wie der römische heres; vielmehr scheint die Tendenz dahingegangen zu sein, den Erben der Liegenschaften, den

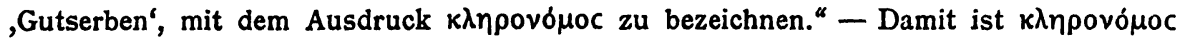
in Mt 21, 38; Mc 12, 7; Lc 20,14 zu vergleichen, wo es auch den Gutserben und

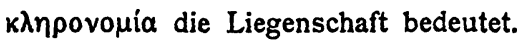

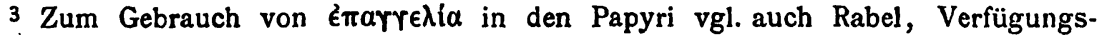
beschränkungen des Verpfänders (1909) S. 102.

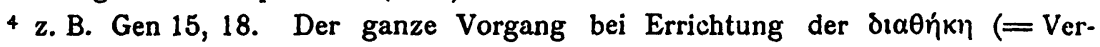

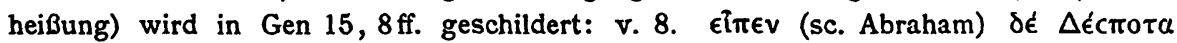

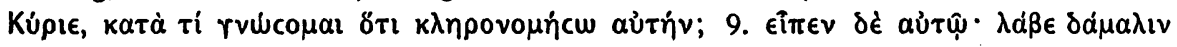

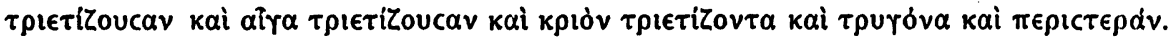

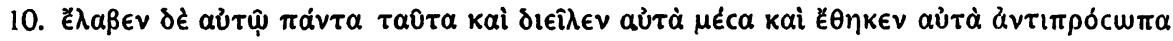

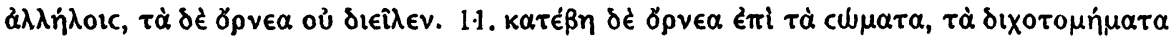

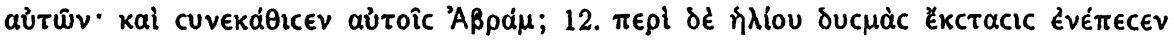

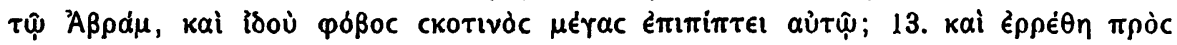

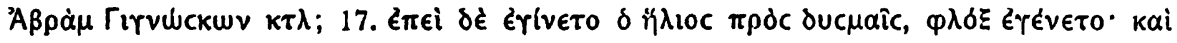

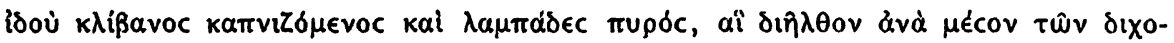

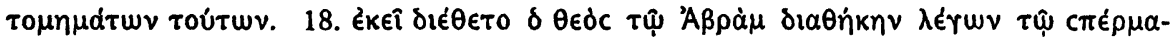

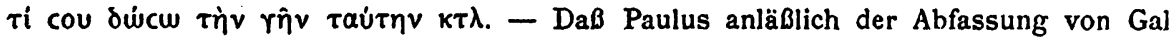
$3,15 \mathrm{ff}$. gerade an diese Stelle (neben anderen, wo sich auch das $k \alpha i$ von $\mathrm{Cal} 3,16$ vor dem $\tau \hat{\varphi}$ c $\pi \epsilon \rho \mu \alpha \tau \imath$ findet, wie z. B. Gen 22,18$)$ denkt, ergibt sich aus dem $\epsilon \rho \rho \in \theta \eta \eta$

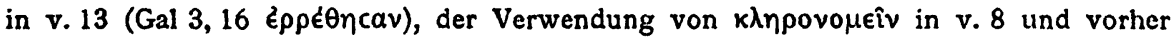
öfter, sowie aus dem Vorkommen von $\delta เ \alpha \theta \dot{\eta} \kappa \eta$; auch ist schon in Gal 3, 6 die Wendung

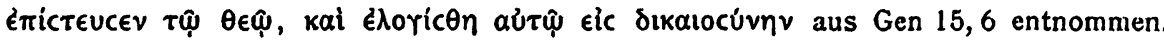

5 Wie es kommt, daß in den LXX an der eben zitierten und an anderen Stellen, wo es sich nicht um ein Rechtsgeschäft von Todeswegen, sondern um ein Geschäft unter Lebenden ohne Beziehung auf den Todesfall des Zuwendenden, und zwar ein 
und - worauf schon dieses Wort führt, unter dem zur Zeit des Paulus nach allgemeinem Sprachgebrauch nur ein Àkt der eben bezeichneten Art verstanden wurde - verbindet damit einen. Vergleich mit der

zweiseitiges Geschäft, einen Vertrag handelt, das Wort $\delta\llcorner a \dot{\eta} \kappa \eta$ als Übersetzung von vẹִּרית verwendet werden konnte, ist hier nicht näher zu untersuchen, da dies für das Verständnis der Paulusstelle nichts austut, für welche hier nur der Sprachgebrauch

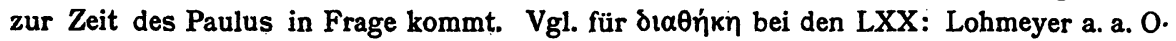
S. $5 \mathrm{ff} .78 \mathrm{ff}$. und die dort Zitierten, außer Mitteis, Grundzüge S. $234 \mathrm{ff}$. namentlich Bruck, Die Schenkung auf den Todesfall im griech. und röm. Recht I. Teil, in Leonhards Studien zur Erläut. d. bürg. Rechts Bd. 31, 1909 (mit Besprechung von Rabel Z. Sav. St. XXX $468 \mathrm{ff}$.), Bruck, Zur Geschichte der Verfügungen von Todes wegen im altgriech. Recht, 1909, insbesondere S. $16 \mathrm{ff}$; weiter Behm, Der Begriff $\delta \imath \alpha \theta \dot{\eta} \kappa \eta$ im Neuen Testament (1912) S. 5 ff. 17 ff. Neuestens: Riggenbach, Der Brief an die Hebräer (Bibl. Zeitu. Streitfragen X. Serie 11./12. Heft) 1916 S. $29 \mathrm{ff}$. - Es trägt zum Verständnis des Gebrauchs von $\delta\llcorner a \theta \dot{\eta} \kappa \eta$ bei den LXX bei, wenn man sich die Geschichte der Verfügungen von Todeswegen im griechischen Recht vergegenwärtigt. Diese haben sich hier (wie auch im römischen Recht) erst allmählich aus zweiseitigen Rechtsgeschäften, Verträgen unter Lebenden entwickelt, nämlich das Adoptionstestament aus der Adoption unter Lebenden, das Vermächtnistestament, als dessen Fortbildung die hellenistische, zur Zeit des Paulus allgemein verbreitete $\delta \iota \theta \dot{\eta} k \eta$ erscheint, aus der Schenkung unter Lebenden auf den Todesfall, ebenfalls einem zweiseitigen Geschäft, einem Vertrag. Diese Entwickelung war zur Zeit der LXX zwar soweit fortgeschritten, daB damals

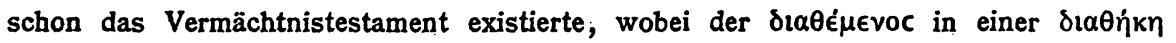
(die sich z. B. in Mitteis, Chrest. 301, v. J. 225 v. Chr., als einseitiges, letztwilliges Rechtsgeschäft darstellt) von Todes wegen sein ganzes Vermögen oder Teile desselben an eine oder mehrere Personen vergab (die aber zu dieser Zeit noch nicht als kinpo-

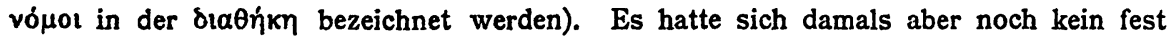
ausgeprägter Sprachgebrauch herausgebildet, wonach unter $\delta i a \theta \dot{\eta} \kappa \eta$ nur ein Rechtsgeschäft von Todeswegen, und zwar der ebenbezeichneten Art zu verstehen gewesen wäre; so läßt sich aus nicht allzu weit vor den LXX liegender Zeit der Gebrauch von $\delta ı a \dot{j} k \eta$ zur Bezeichnung von Rechtsgeschäften unter Lebenden ohne Beziehung auf einen Todesfall, und zwar von zweiseitigen Geschäften, Verträgen belegen. Namentlich ist aber in dieser Zeit die Verwendung des Verbs $\delta \iota \alpha i \theta \in c \theta \alpha \imath$ für derartige Ge-

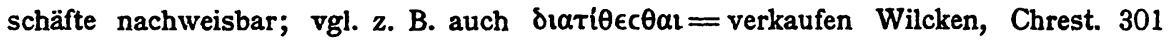
(ca. 241/210 v. C.); zu beachten ist auch Digesten 47, 22, 3 (de colleg. et corporibus)

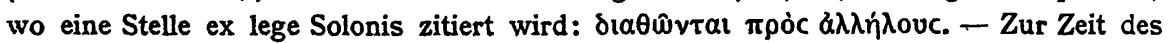
Paulus hatte sich die hellenistische $\delta \alpha \theta \dot{\eta} \kappa \eta$ in jahrhundertelangem Gebrauch als erb-

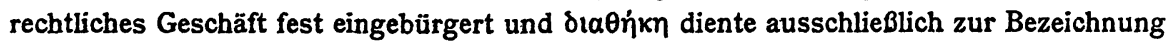
des im Text näher charakterisierten Rechtsgeschäfts von Todeswegen. - Wenn man dazu 2. B. die aus $117-137 \mathrm{n}$. Chr. stammende, hellenistische $\delta\llcorner a \theta \eta \dot{k} \eta$ bei Mitteis, Chrest. 303 vergleicht, so ergeben sich noch zwei weitere Merkmale: Sie ist ein einseitiges Rechtsgeschäft, da sie nur die Willenserklärung des $\delta \_a \in$ é $\mu \in v o c$ (vor der Behörde und vor Zeugen) enthält; eine Mitwirkung der Bedachten findet nicht statt. Sie ist ferner eine letztwillige Verfügung, d. h. der jeweils letzte Wille gilt, so daß die

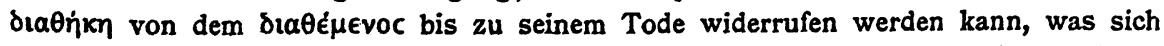

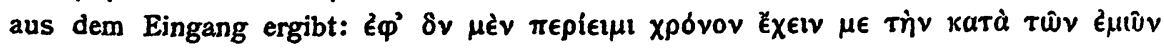


menschlichen $\delta \iota \alpha \theta \dot{\eta} \kappa \eta$. Dieser Vergleich wird allerdings hier nur hinsichtlich eines Punktes ausdrücklich durchgeführt; nämlich hinsichtlich der Unantastbarkeit der göttlichen und der menschlichen $\delta \iota \alpha \theta \dot{\eta} \kappa \eta$ durch Dritte.

Wie sich Paulus das Verhältnis der göttlichen zur menschlichen $\delta ı \alpha \eta^{\prime} \kappa \eta$ im übrigen denkt, ist hier nicht zu untersuchen. Nur ist darauf hinzuweisen, wie Paulus durch die Tatsache, daß - und die Art, wie

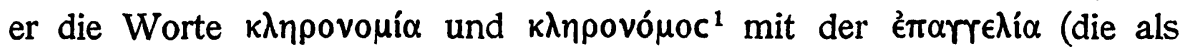
$\delta \imath \alpha \theta \dot{\eta} \kappa \eta$ vorher beżeichnet wurde) verbindet, die Erinnerung an die bei der - vorher erwähnten - menschlichen $\delta \prec \alpha \eta \dot{k n}$ bestehenden Ver-

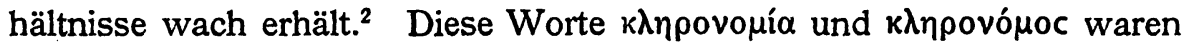

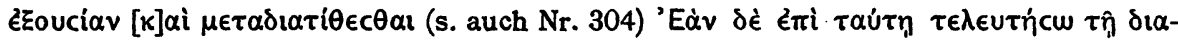

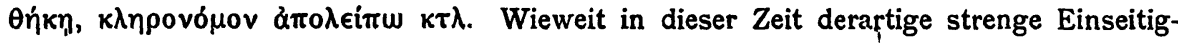
keit und Widerruflichkeit bei einer Verfügung von Todeswegen voihanden sein müssen, damit diese nach dem damaligen Sprachgebrauch als $\delta \iota \alpha \dot{\eta} \kappa \eta$ bezeichnet werden kann, werden erst künftige Untersuchungen über die hellenistische $\delta \iota \alpha \theta \dot{\eta} \kappa \eta$ auf Grund des gesamten Materials klarstellen.

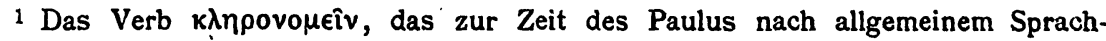
gebrauch den Erwerb im Wege des Erbgangs (auf Grund von Gesetz oder $\delta \iota \alpha \theta \dot{\eta} k \eta$ ) bezeichnet, findet sich bei den LXX in Verbindung mit der Verheißung an Abraham

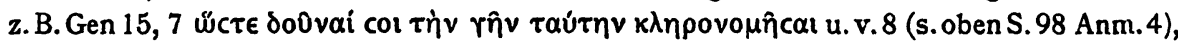

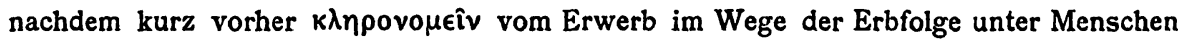

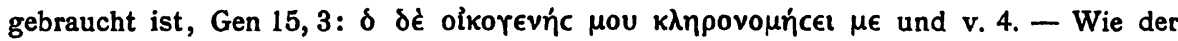
Gebrauch von $\kappa \lambda \eta \rho o v o \mu \in \hat{\imath} v$ in Gen 15, 7 u. 8 und in sonstigen Fällen, wo an den Erwerb im Wege des Erbgangs nicht gedacht ist (s. darüber Lohmeyer a. a. O. S. $97 \mathrm{ff}$.), zu erklären ist, bedarf hier nicht der Untersuchung, da es für unsere Zwecke genügt,

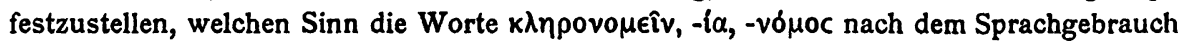
zur Zeit des Paulus hatten. - Bei einer Untersuchung des Gebrauchs von kinpovoutîv durch die 70 Dolmetscher wäre wohl auch die Frage zu prüfen, wie weit viel-

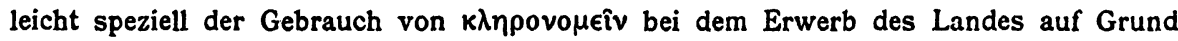
der VerheiBung in Zusammenhang stehen könnte mit der Verteilung von erobertem Land in einzelnen $k \lambda \hat{n} \rho o l$, wie wir dies ja von den Ptolemäern aus der Zeit der LXX aus den Papyri kennen. Siehe Wilcken, Grundz. S. $280 \mathrm{ff}$; vgl. z. B. Jos 12, 7, wo es bei der Verteilung des eroberten Landes an die Stämme Israels durch Josua heißt:

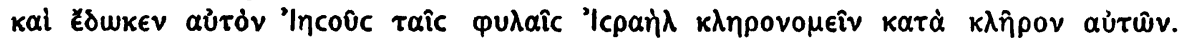

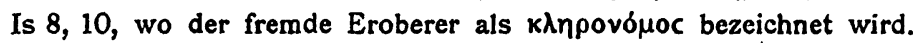

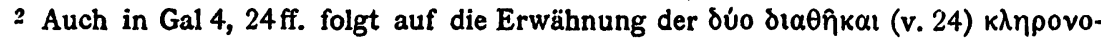
$\mu \eta \dot{c \in l}$ ( $v$. 30 im alttestamentlichen Zitat). - In den freikombinierten alttestamentlichen Stellen Röm 11, 27 könnte vielleicht an eine Einwirkung des Gedankens an die menschliche $\delta\llcorner\alpha \theta \dot{\eta} \kappa \eta$ insofern gedacht werden, als hier die Sündenvergebung als Inhalt der göttlichen $\delta \iota \alpha \theta \dot{k} \kappa \eta$ angegeben ist. Darf man dabei an die Parallele: Sündenvergebung = Schulderlaß denken (Deißmann, Paulus S. 99), so ist ein Berührungspunkt dadurch gegeben, daß auch in der menschlichen $\delta\llcorner\alpha \dot{\eta} \kappa \eta$ eine Schuld erlassen werden kann (vgl. ă

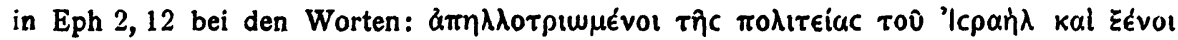


den Lesern des Briefs aus dem täglichen Leben und dem Rechtsverkehr in der Bedeutung von Erbschaft und Erben (auf Grund einer

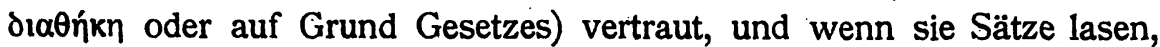
in welchen im Zusammenhang mit $\delta\llcorner a \theta \dot{\eta} \kappa \eta$ die Rede war von $k \lambda \eta \rho o-$

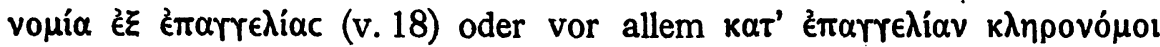
(v. 29), so wurden sie an die häufig gebrauchten Wendungen erinnert

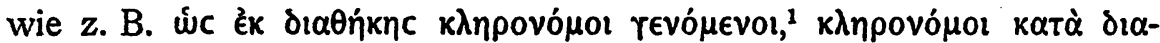

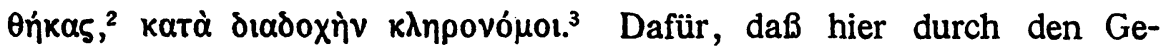

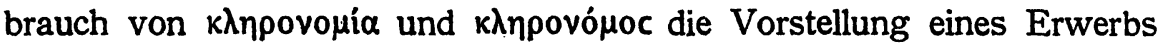

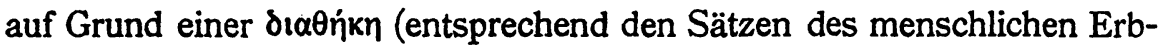
rechts) bei seinen Lesern hervorgerufen wurde und daß dies von Paulus gewußt und gewollt war, spricht auch, daß in dem sofort folgenden Abschnitt in Gal 4,1 bei einem neuen, direkten Hinweis auf Sätze und

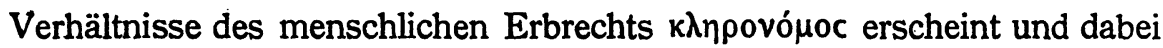
auch - wenn auch ohne ausdrückliche Erwähnung derselben - auf die menschliche $\delta \iota \theta \hat{\eta} \kappa \eta$ Bezug genommen wird, in welcher sich die Bestimmung des Termins für die Beendigung der Vormundschaft $(\pi \rho 0-$

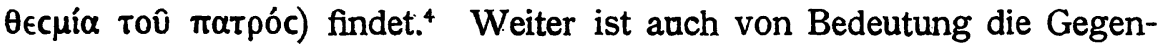

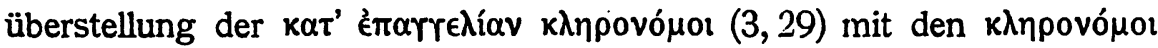

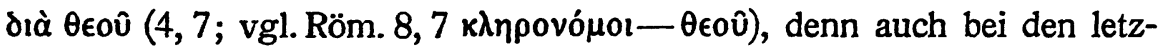
teren Stellen handelt es sich um die - wenn auch nur stillschweigende -

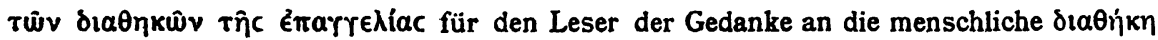
nicht allzufern lag und daB sich auch der Verfasser des Briefs dieses Umstands bewußt war, ergibt folgende Oberlegung: Mit den Worten Żévoc und $\pi 0 \lambda \iota \tau \epsilon l \alpha$ verband der Leser zur damaligen Zeit in erster Linie die Vorstellung von den Zuständen im römischen Reich, in dem er selbst nur als Év́oc (= Peregrine; diese bildeten ja da-

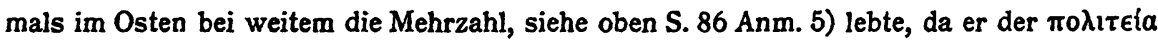
'Punaiwr (s. Apg. 22, 28, Constit. Antonina Mitteis, Chrest. 377 1. 7/8) nicht teilhaftig war. In dieser Auffassung des Wortes Éंvoc wurde der Leser noch bestärkt,

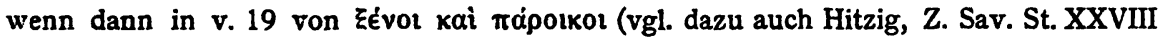
S. 212 ff.) im Gegensatz zu cuviodĩal die Rede war. Unter diesen Umständen rief

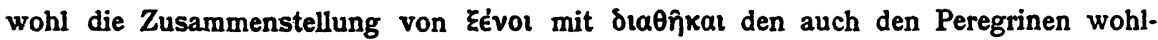
bekannten Satz des römischen Rechts ins Gedächtnis, daß der छ́́voc (Peregrine) auf Grund eines römischen Testaments ( $(1 \alpha \theta \dot{\eta} \kappa \eta)$ nicht Erbe (heres, $k \lambda \eta \rho o v \delta \mu o c)$ werden kann.

1 Mitteis, Chrest. 89, 30.

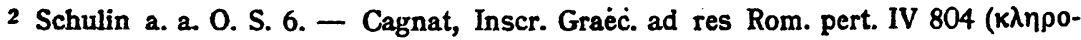

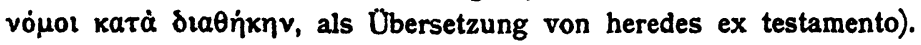

3 = gesetzliche Erben, Kreller a. a. O. S. 56. Die gesetzlichen Erben werden

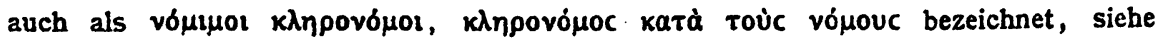
Kreller a. a. O., wie auch die Verbindung katà toùc vómouc kai katà bıäńkac sich findet.

4 Siehe unten S. $105 \mathrm{f}$. 
Bezugnahme auf den Satz des menschlichen Erbrechts, wonach der Adoptivsohn Erbe ( $\kappa \lambda \eta \rho o v o ́ \mu o c)$ des Adoptivvaters ist. ${ }^{1}$

Bei seinem Bestreben, darzutun, daß die an Abraham und seinen

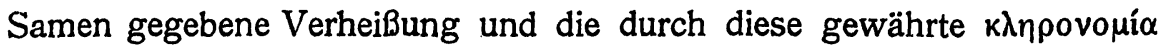
nicht durch das später erlassene Gesetz berührt wird, bot sich Paulus bei einem Vergleich der Verheißung als göttlicher $\delta\llcorner\alpha \theta \dot{\eta} \kappa \eta$ mit der menschlichen $\delta ı \theta \emptyset$ in dem - vermutlich in Anlehnung an ein Testamentsformular gebildeten allgemein bekannten und geläufigen Satz dar: daß keiner (d. h. kein Dritter) die gültige $\delta \iota \alpha \dot{\eta} \kappa \eta$ eines Menschen kraftlos machen oder über den Nachlaß verfügen kann. Aus diesem Satz des menschlichen Testa-

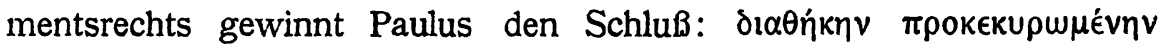

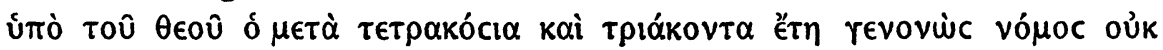

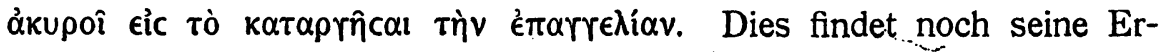
läuterung in v. 19.

Es wird aber noch eine Ausführung in v. $18^{2}$ dazwischen geschoben, die sich ganz ähnlich Röm 4,13.14 ${ }^{3}$ findet und erst im Zusammenhang mit dieser und anderen Stellen des Römerbriefs ganz verständlich ist. V.18 ist wohl dahin zu verstehen, daß das Gesetz nicht die Ge-

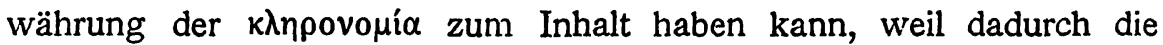

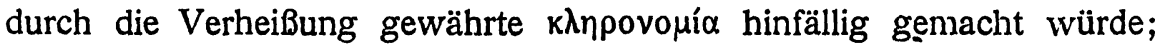

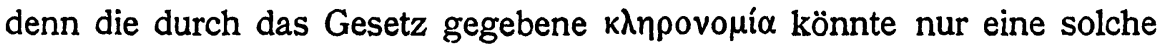

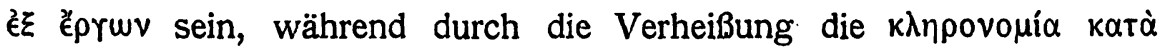

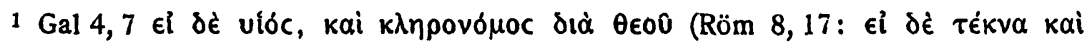

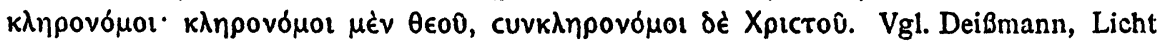

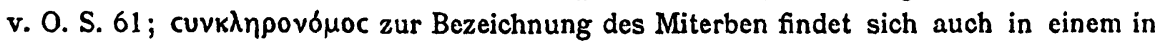
den Digesten enthaltenen Auszug aus einem Testament, siehe Kübler, Z. Sav. St. XXVIII S. 194). - Anders Lohmeyer a. a. O. S. $140 \mathrm{ff}$. Wenn Lohmeyer S. $142^{2}$ sagt: „Es ist zu beachten, daB, will man auf Grund der rechtlichen Bedeutung von dıaӨjikn und $\kappa \lambda \eta p o v o \mu \epsilon \hat{v}$ eine religiöse Verbindung $z$ wischen ihnen herstellen, dies konsequenterweise auch für die $\pi \alpha \lambda \alpha ı \dot{\alpha} \delta \iota \alpha \theta \dot{\eta} \kappa \eta$ geschehen müsse, welche für Paulus den Charakter des Gesetzes trägt (s. Gal 4, 24; 2 Kor 3, 14); und doch wird von Paulus diese Verbindung Röm 4, 14, Gal 3, 18 aufs schärfste geleugnet" - so ist dagegen zu sagen, daß Paulus gerade an unserer Stelle, wo er $\delta$ ta $\theta \dot{n} k \eta$ und $k \lambda \eta \rho o v o \mu l \alpha$ miteinander in Verbindung bringt, das Gesetz nicht $\delta \alpha \theta \dot{\eta} \kappa \eta$ nennt, gerade um den Schluß: $v \delta \mu \rho c=\delta i \alpha \theta \dot{\eta} \kappa \eta$, also Ernennung von $\kappa \lambda \eta p o v o ́ \mu o l$ zu vermeiden.

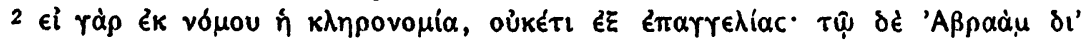

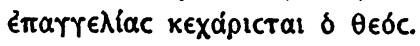

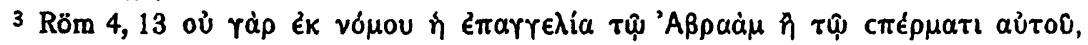

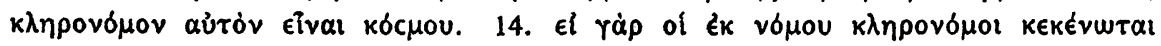

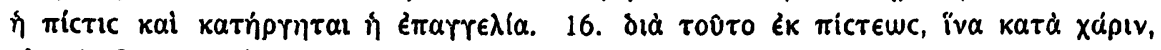

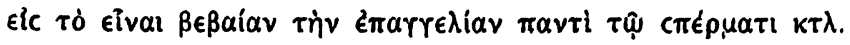




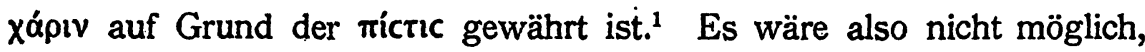

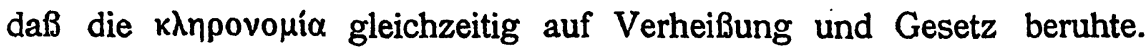
Es gäbe nur ein entweder - oder, wobei aber das Gesetz zurücktreten müßte, weil es sich in diesem Falle als ein unzulässiges émı eine Verfügung über die Erbschaft durch einen Dritten darstellen würde. -

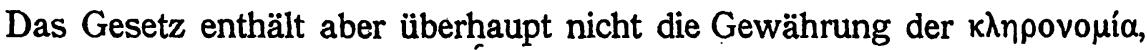
es hat keinen erbrechtlichen Inhalt, ist kein Erbgesetz, sondern es ist nach v. 19 Tŵv $\pi \alpha \rho \alpha \beta a ́ c \epsilon \omega v ~ \chi \alpha ́ p ı v^{2}$ zugefügt und überdies nur von zeit-

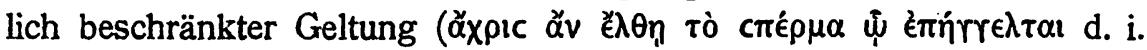
Christus).

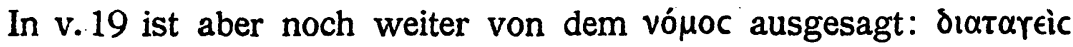
$\delta \imath^{\prime}$ ảrrétuwv. Das ist wohl dahin zu verstehen, daß das Gesetz erlassen ist $^{3}$ - nicht unmittelbar von Gott, sondern - durch Engel. ${ }^{4}$ Dies würde die Heranziehung des Satzes aus dem menschlichen Testamentsrecht erläutern, daß keiner (wobei an den $\delta i \alpha \theta \dot{\epsilon} \mu \in v o c$ nicht gedacht ist) die $\delta \alpha \theta \dot{\eta} k \eta$ kraftlos machen oder durch Verfügungen beeinträchtigen kann - denn dann ist eben der vónoc nicht unmittelbar von Gott

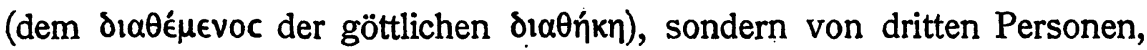
den Engeln, erlassen und kann deshalb (ganz abgesehen von seinem

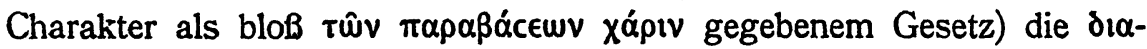
$\theta \dot{n} k \eta$ Gottes nicht beinflussen (weder im Sinne des $\alpha \dot{\theta} \theta \tau \in \hat{\imath} v$ noch in dem

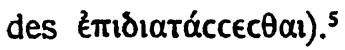

Gegen diese Auffassung läßt sich anführen, daß das Gesetz von Paulus selbst an anderer Stelle ausdrücklich als vómoc rov̂ $\theta \in 0 \hat{~ b e-~}$

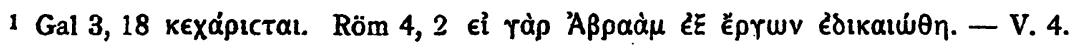

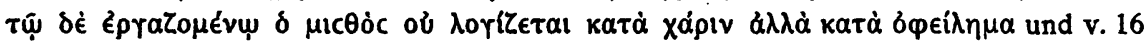
(s. vorige Anm.).

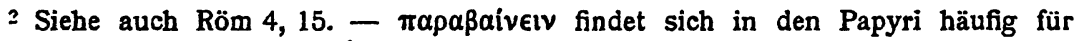
Übertretungen von Vertragsbestimmungen und in Strafklauseln, auch bei Testamenten, siehe Berger a. a. O.S. 3. - Von der Ubertretung ediktaler Vorschriften eines praefectus Aegypti wird es gebraucht im Edikt Mitteis, Chrest. 188 (12 n. C.) Col. III Z. 11 ff.:

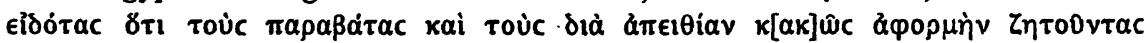

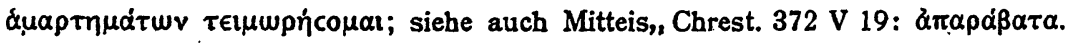

$3 \mathrm{Zu} \delta$ datácceiv s. oben S. 93 Anm. 2 und Hesiod Ěpr. 274.

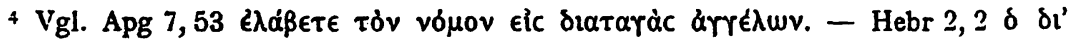

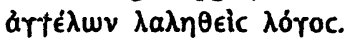

5 Nimmt man an, daß nicht die Engel als Erlasser des Gesetzes zu betrachten sind, so muß man, um diese Anwendung des Satzes aus dem menschlichen Testamentsrecht, wonach kein Dritter die $\delta \alpha \theta \dot{\eta} \kappa \eta$ entkräften oder über die Erbschaft ver-

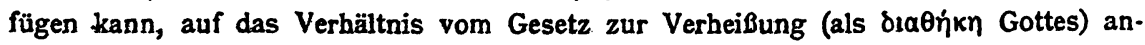
wenden zu können, annehmen, daß der personifizierte vónoc als selbständige Größe, als Dritter, Gott gegenübersteht. 
zeichnet wird $^{1}$ - wogegen aber hinwiederum $\mathrm{zu}$ beachten ist, daß an unserer Stelle diese Bezeichnung augenscheinlich absichtlich vermieden

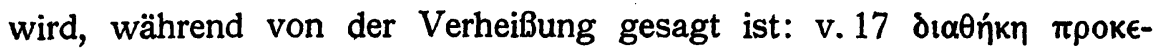

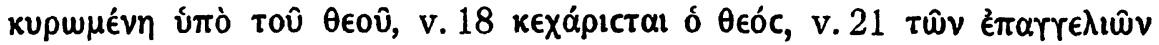

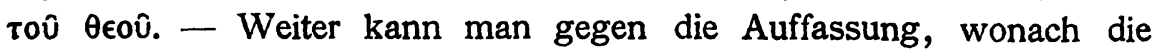
Engel das Gesetz erlassen haben, geltend machen, daß es diatareic dió und nicht inó heißt. Jedoch wird auf den Gebrauch der Präposition oró kein so entscheidendes Gewicht gelegt werden dürfen, da sie ja auch den Urheber bezeichnen kann. ${ }^{2}$ Aber auch wenn man oló hier instrumental nimmt, so ist anzunehmen, daß Paulus damit nur andeuten wollte, daß zwar mittelbar das Gesetz auf Gott zurückgeht, daß aber doch in erster Linie als die das Gesetz Erlassenden die Engel, also Dritte, zu betrachten sind. Schließlich dürfte auch die Erwähnung des $\mu \in c i ́ t \eta c$

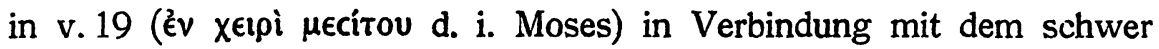

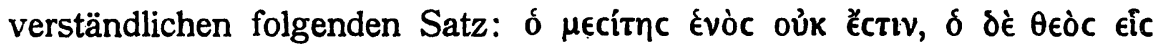
éctiv für die Ansicht sprechen, daß die Engel nach Auffassung des Paulus das Gesetz erlassen haben. Allerdings ist eine sichere. Deutung dieses Satzes wohl kaum möglich. Immerhin ist es das Wahrschein-

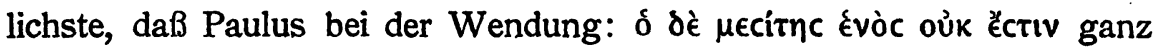
allgemein an die Figur des $\mu \in c i ́ t \eta c$ denkt, die im Rechtsleben der damaligen Zeit sehr häufig begegnete, und daß er damit das (seinen Lesern bekannte) all den verschiedenen Spielarten ${ }^{3}$ des $\mu \in c i t \eta c$ Gemeinsame betonen will, daß nämlich, wo ein $\mu \in c i t \eta c$ in die Erscheinung tritt, immer mehrere Personen beteiligt sind, für die der $\mu \in c i ́ t \eta c$ tätig ist. Wo ist nun hier in unserem. Fall diese Mehrheit zu suchen? Da durch ó $\theta$ eòc eíc éctiv doch wohl Gott nicht nur als allein handelnde Person, sondern auch als nur eine von mehreren Parteien (wobei auf der anderen Seite das Volk Israel $z u$ denken wäre) ausgeschieden und darauf hingewiesen werden soll, daß der $\mu$ ecítnc nur für andere Personen tätig gewesen ist - so ist es das Nächstliegende, die kurz vorher erwähnten ărre

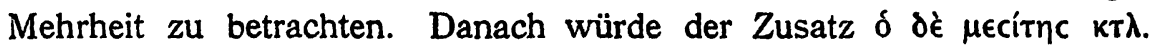
gemacht sein, um auf Grund einer allerdings ganz an der Oberfläche

1 Röm 7, 22. 25.

2 In den Papyri bringt dıá zwar überwiegend, aber doch nicht stets Stellvertretung oder Botendienst zum Ausdruck, vgl. Wenger, Die Stellvertretung im Rechte des Papyri (1906) S. 9 ff.

3 Siehe Mitteis, Hermes XXX (1895) S. 616 ff. Für unsere Stelle schließt sich Mitteis a.a. O. der von der hier vertretenen abweichenden Auffassung des $\mu \in c i \tau \eta c$ von Halmel an. 
haftenden Beobachtung hinsichtlich des $\mu \in c i t \eta n c$ im allgemeinen, aus dem Auftreten eines $\mu \in c i ́ t \eta c$ bei dem Erlaß des Gesetzes eine Begründung für die Ansicht zu gewinnen, daß nicht einer (Gott), sondern eine Mehrheit (die Engel) das Gesetz erlassen hat. Wie sich dabei

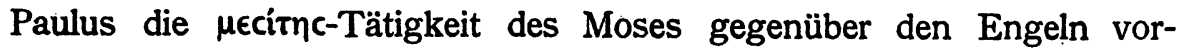
gestellt hat, bleibt angesichts der unbestimmten Ausdrucksweise des Paulus und der Vieldeutigkeit von $\mu \in c i t \eta c$ ganz im unklaren.

Ebenfalls dem Gebiet des Erbrechts und auch noch demjenigen des Vormundschaftsrechts gehört die an die eben behandelte direkt anschließende Stelle Gal 4,1.2 an, und man kann mit großer Wahrscheinlichkeit die Gedankengänge aufdecken, die zur Entstehung dieser Stelle führten. Durch den Vergleich der göttlichen mit der menschlichen $\delta \iota \alpha \theta \dot{\eta} k \eta$ waren die Gedanken des Paulus auf die letztere gelenkt

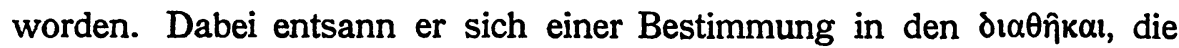
ihm wohl schon selbst im täglichen Leben häufig begegnet war, wonach der Vater seinem unmündigen Sohn Vormünder bestellt und einen Termin festsetzt, bis $\mathrm{zu}$ welchem-die Vormundschaft dauern sollte, und es schienen ihm die hierbei in Frage kommenden Lebensund Rechtsverhältnisse für seine Beweiszwecke geeignet. So sagt er denn:

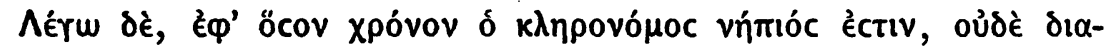

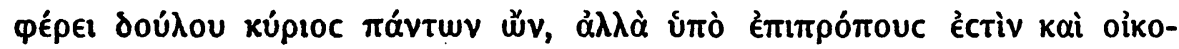
vó

Allerdings hat man daran gezweifelt, daß es sich hier um eine testamentarische Bestimmung des Endtermins einer Vormundschaft handle, und hat vielmehr mitunter geglaubt, daß der Vater lebend gedacht sei. Diese Ansicht scheitert zwar nicht schon an dem Wort

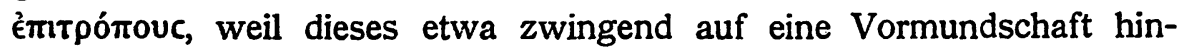

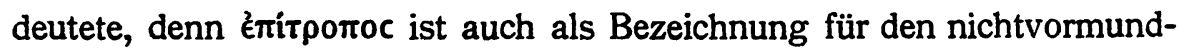
schaftlichen Verwalter belegt. ${ }^{1}$ Es spricht aber doch alles - insbeson-

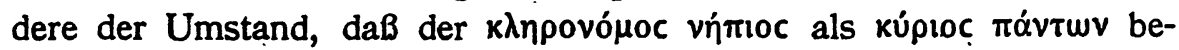
zeichnet wird, ferner, daß kurz vorher von $\delta\llcorner\alpha \theta \dot{\eta} \kappa \eta$ die Rede ist, und daß, wie uns jetzt die Papyri zeigen, Bestimmungen des Vaters über

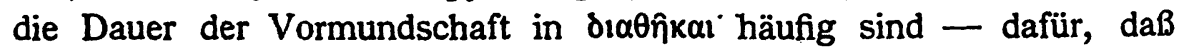
die Stelle folgendermaßen verstanden werden muß: Für den unmün-

1 Vgl. z. B. Oxy III 501 (187 n. C.), Wenger, Stellvertretung S. $228^{1}$, B. G.U. 106

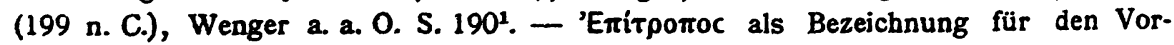

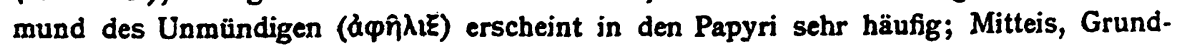
züge S. 248. 
digen $^{1}$ Sohn $^{2}$ sind im Testament des Vaters mehrere Vormünder ernannt worden, welche bis $\mathrm{zu}$ dem im väterlichen Testament bezeichneten Endtermin die Vormundschaft führen. $\mathrm{Da}$ während dieser Zeit die Verwaltung des Vermögens, obwohl der Unmündige dessen „Herr"3

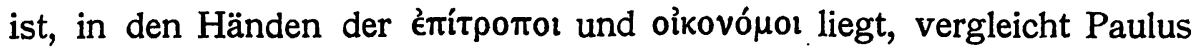
die Lage des Unmündigen mit der eines Sklaven. ${ }^{4}$

Auch hier taucht die Frage auf, ob Paulus Sätze einer bestimmten Rechtsordnung im Auge hat, und eventuell, welche dies ist. Halmel $^{5}$ hat sich auch hier für römisches Recht entschieden; doch verfährt er - wenig gründlich, so daß er dabei auch ohne weiteres - wie in den émí$\tau \rho 0 \pi 0$ r römisch-rechtliche tutores - in den oỉovómol curatores erblickt. Er übergeht dabei die Schwierigkeit, die sich daraus ergibt, daß hier

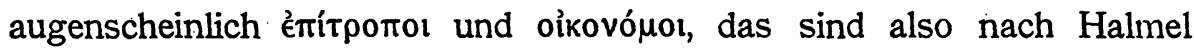
tutores und curatores, für die Zeit der Unmündigkeit $^{6}$ gleichzeitig nebeneinander fungieren sollen, während man doch zunächst hier an eine cura minoris, die sich erst nach erreichter Mündigkeit an die tutela impuberis anschließt, zu denken geneigt ist. Zwar könnte man versuchen, diesem Bedenken damit aus dem Wege $z u$ gehen, daß man annimmt, daß curatores impuberis gemeint seien, die nach den römischen Rechtsquellen mitunter dem tutor während der Unmündigkeit des Mündels adjungiert sein können. ${ }^{7}$ Aber ein solcher adjungierter curator impuberis

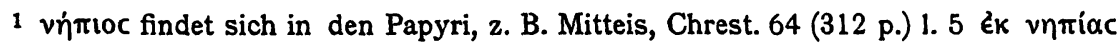

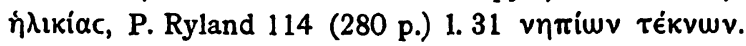

2 eines begüterten Mannes; das kann man aus dem Vorhandensein mehrerer $\epsilon \pi i \tau p o \pi o r$ und olkovómor schließen.

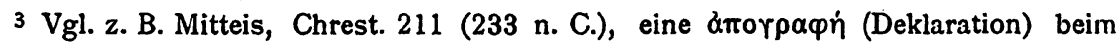
Grundbuchamt (der $\beta \imath \beta \lambda \iota 0 \theta \dot{\eta} \kappa \eta$ É $\gamma \kappa \tau \eta \dot{c \epsilon} \omega v$ ), wo in dem Erledigungsvermerk der Grund-

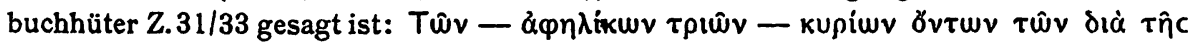

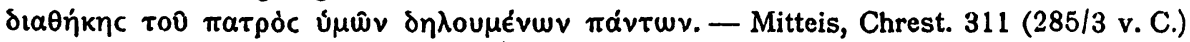

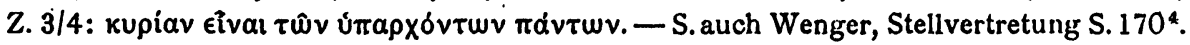

4 Der Sklave steht unter einem Herrn, seinem kúpıoc (vgl. z. B. die königliche Verordnung die Sklaven betreffend aus dem 3.Jahrh.v.C., Mitteis, Chrest. 369). - Über die Frage der Vermögensfähigkeit des gräko-ägyptischen Sklaven siehe Mitteis, Grundzüge 238, Wenger Stellvertr. S. 1677.

5 a. a. O. S. 26 ff. - Vgl. auch Sieffert in Meyers Kom. (9. Auf.) S. 232 ff., ders., Das Recht im NT. S. 14 ff., Conrat a. a. O. S. $221 \mathrm{ff}$.

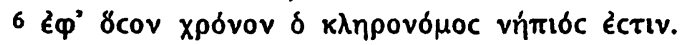

7 Vgl. Taubenschlag, Vormundschaftsrechtl. Studien (1913) S. $47 \mathrm{ff}$. Auch testamentarische Kuratoren begegnen in den Quellen, die aber konfirmiert werden müssen. (Taubenschlag S. 48, 2.) Für einen Zusammenhang zwischen dem oỉovómoc und dem adjungierten curator könnte man darauf hinweisen, daB Pomponius D. 26, 1. 13 pr. von letzterem sagt: qui magis administrator rerum quain curator esse intellegitur, dazu Taubenschlag S. 48 Anm. 7. 
wird erst nach der Zeit des Paulus zum erstenmal von einem römischen Juristen erwähnt; er erscheint in den römischen Quellen außerdem nur in Ausnahmefällen, während Paulus die oikovópor als ganz regelmäßig vorhanden hinstellt - und überdies gibt es meines Wissens zurzeit auch keinen Beleg dafür, daß oỉkovómoc zur Übersetzung von curator verwandt worden ist. ${ }^{1}$

Jedoch erübrigt es sich, auf diese und andere Fragen, die bei einer Heranziehung des römischen Rechts sich ergeben, näher einzugehen, weil es viel mehr Wahrscheinlichkeit für sich hat, daß auch hier Paulus Verhältnisse im Auge hat, die durch eine andere Rechtsordnung geregelt sind. Auch auf dem Gebiet des Vormundschaftsrechts hat - ebenso wie auf dem des Erbrechts - das römische Recht zur damaligen Zeit im römischen Reich nur für römische Bürger gegolten, während für den Peregrinen sein Volksrecht maßgebend war. ${ }^{2}$ So spricht schon der Umstand, daß in Gal 3,15ff. Paulus, wie oben darzutun versucht wurde, nicht das römische testamentum, sondern die hellenistische $\delta\left\llcorner\alpha \theta \dot{\eta} \kappa \eta\right.$ im Auge hat, dafür, daß auch hier hellenistisches ${ }^{3}$ Recht in Frage kommt. Diese Annahme erhält eine starke Stütze dadurch, daß sich in hellenistischen ठı $\alpha \theta \hat{\eta} k \alpha i$ auf Papyrus Anordnungen über die Dauer der Vormundschaft finden, entsprechend der $\pi \rho 0 \theta \epsilon c \mu i \alpha$

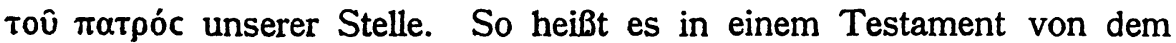

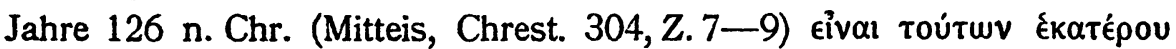

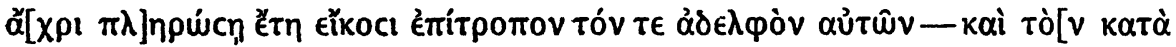

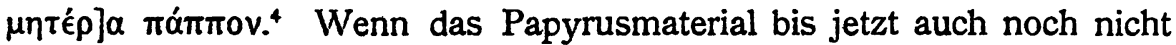

1 Uber die Terminologie der Vormundschaft in den Papyri vgl. Mitteis, Grundzüge $248 \mathrm{ff}$.

2 Mitteis, Reichsrecht S. 107/108. - Vgl. aber auch Mitteis, Chrest. 304 (126 n. C.) Einleitung und Grundz. 251 Anm. 4 (über Oxy 487, Chrest. 322, v. J. 156 n. C.) über zwei Fälle, in denen gelegentlich die römische cura minorum bis zum 25. Jahr auf Peregrinen übertragen sein könnte; doch ist der Sachverhalt sehr zweifelhaft. Ubrigens

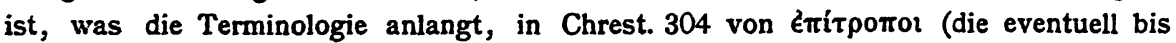

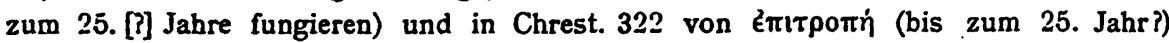
die Rede.

3 Gegen die Heranziehung des galatischen Rechts (die Sieffert, Das Recht im NT S. $14 \mathrm{ff}$. und in Meyer, Kom. S. 233/234, ebenso auch Conrat a. a. O. S. $212 \mathrm{ff}$. für möglich hält) und des jüdischen Rechts siehe die oben S. 85 f. angeführten Gründe.

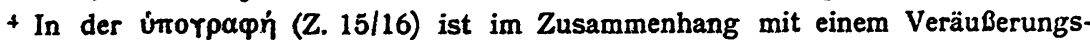

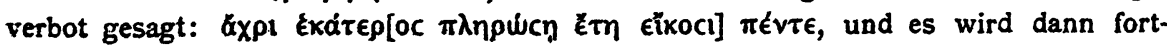

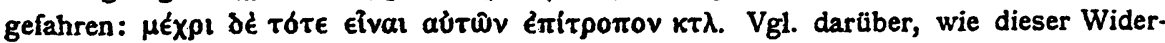
spruch zu lösen ist, Mitteis in der Einleitung zu Chrest. 304. Das Alter von 20 Jahren erscheint als Endtermin der Fürsorge seitens der Vatersschwester auch in Oxy 495 (181-189 n. C.). 
erlaubt; 'festzustellen, welche Regeln im einzelnen für die Bestimmung des Eintrittes der Mündigkeit und die Beendigung der Vormundschaft bei den Griechen in Ägypten bestanden, ${ }^{1}$ so zeigen uns die Papyri doch eine Gewohnheit, in der väterlichen $\delta \iota \hat{\theta} \eta \dot{n} \kappa \eta$ bei Anordnung der Vormundschaft auch eine Klausel über deren Beendigung aufzunehmen, die wohl auch sonst in den $\delta \_\theta \hat{\eta}$ kal des hellenistischen Rechtskreises

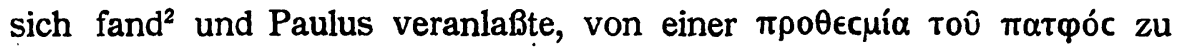
sprechen.

Die Ernennung eines Vormunds (oder mehrer émítpotoı) für den Unmündigen im väterlichen Testament, an welche Paulus, wie aus der Erwähnung der $\pi \rho 0 \theta \epsilon c \mu i \alpha$ zu schließen ist, hier denkt, ist ja im griechischen Rechtskreis schon seit alter. Zeit üblich gewesen, ${ }^{3}$ und sie be-

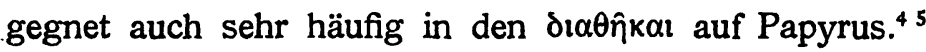

Für eine nähere Bestimmung des Charakters der oikovó $\mu 0$ bieten die Papyri bis jetzt keinen weiteren Anhalt. Es sind damit wohl unter Aufsicht der émítponor tätige. Vermögensverwalter gemeint, die man sich als von dem Vater noch zu seinen Lebzeiten oder von den émi$\tau \rho \circ \pi 01^{6}$ bestellt wird denken müssen, wenn auch der Zusammenhang den Gedanken nahe legt, daß sie auch im Testament des Vaters mit

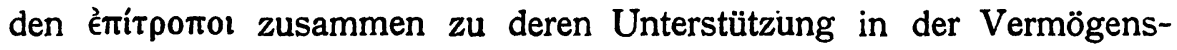
verwaltung bestimmt sein könnten.

1 Vgl. Mitteis Grundz. 251. „Ja, man kann überhaupt zweifeln, ob die Altersgrenze eine fixierte war." (Vgl. dazu jetzt noch Taubenschlag, Z. Sav. St. XXXVII 199ff.)

2 Schon der Umstand, daß der Volljährigkeitstermin bei den Griechen statutarisch verschieden geregelt war (Mitteis, Grundz. 251), gab wohl Veranlassung dazu, daß der Vater ihn - wenn auch in Öbereinstimmung mit dem gesetzlichen Termin seines Heimatsstaats - in seiner $\delta \prec \theta \dot{\eta} \kappa \eta$ bezeichnete.

3 Vgl. Meyer-Schömann-Lipsius a. a. O. II 532, auch das Testament des Aristoteles, Z. Sav. St. I S. 20.

4 So in der eben mitgeteilten Stelle aus Mitteis, Chrest. 304; siche auch Wenger, Stellvertretung S. $236^{2}$, P. Ryland 153 (138-161 p) 1. $18 / 19$.

5 Über die Verwaltung des Vermögens durch den $6 \pi i \tau \rho o \pi \circ$ nach dem Rechte der Papyri s. Mitteis, Grundz. S. 249. 258.

$6 \mathrm{Vgl}$. dazu die Fälle, in welchen in den Papyri für einen $\alpha \varphi \hat{\eta} \lambda ı \xi$ ein

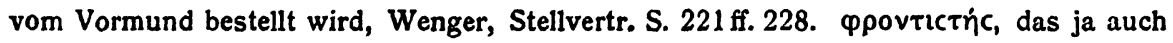
den Verwalter im allgemeinen bezeichnet (Wenger S. 13), begegnet in den Papyri häufig für den Vertreter beim AbschluB eines Rechtsgeschäfts, und zwar auch bei Unmündigen (s. z. B. Wenger S. 240), doch bezeichnet es beim $\measuredangle \varphi \hat{\eta} \lambda \iota \xi$ wohl oft nicht den

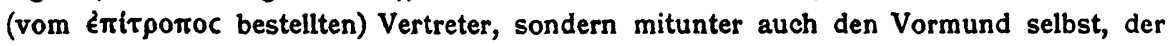
in seiner Eigenschaft als Vermögensverwalter seines Mündels als dessen Vertreter - handelt, Mitteis, Grundz. 249/250. 